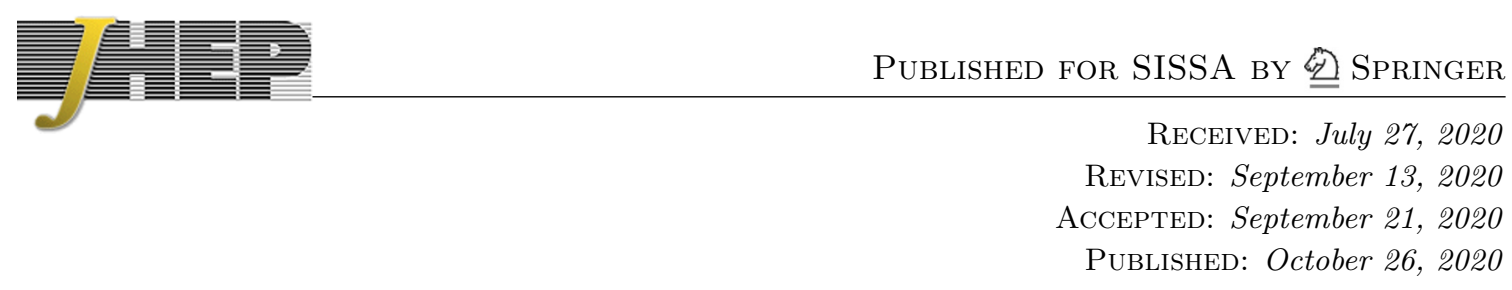

\title{
JT supergravity and Brezin-Gross-Witten tau-function
}

\author{
Kazumi Okuyama $^{a}$ and Kazuhiro Sakai ${ }^{b}$ \\ ${ }^{a}$ Department of Physics, Shinshu University, \\ 3-1-1 Asahi, Matsumoto 390-8621, Japan \\ ${ }^{b}$ Institute of Physics, Meiji Gakuin University, \\ 1518 Kamikurata-cho, Totsuka-ku, Yokohama 244-8539, Japan \\ E-mail: kazumi@azusa.shinshu-u.ac.jp, kzhrsakai@gmail.com
}

ABSTRACT: We study thermal correlation functions of Jackiw-Teitelboim (JT) supergravity. We focus on the case of JT supergravity on orientable surfaces without time-reversal symmetry. As shown by Stanford and Witten recently, the path integral amounts to the computation of the volume of the moduli space of super Riemann surfaces, which is characterized by the Brezin-Gross-Witten (BGW) tau-function of the KdV hierarchy. We find that the matrix model of JT supergravity is a special case of the BGW model with infinite number of couplings turned on in a specific way, by analogy with the relation between bosonic JT gravity and the Kontsevich-Witten (KW) model. We compute the genus expansion of the one-point function of JT supergravity and study its low-temperature behavior. In particular, we propose a non-perturbative completion of the one-point function in the Bessel case where all couplings in the BGW model are set to zero. We also investigate the free energy and correlators when the Ramond-Ramond flux is large. We find that by defining a suitable basis higher genus free energies are written exactly in the same form as those of the KW model, up to the constant terms coming from the volume of the unitary group. This implies that the constitutive relation of the KW model is universal to the tau-function of the KdV hierarchy.

KEYwords: 2D Gravity, Integrable Hierarchies, Matrix Models

ARXIV EPRINT: 2007.09606 


\section{Contents}

1 Introduction 1

$2 \quad$ JT supergravity and Brezin-Gross-Witten $\tau$-function 2

3 Free energy of Brezin-Gross-Witten model $\quad 6$

$\begin{array}{lll}3.1 \text { Cut-and-join operator } & 6\end{array}$

3.2 String equation 8

3.3 Free fermion representation of BGW $\tau$-function $\quad 9$

$\begin{array}{lll}3.4 & \text { Large genus asymptotics } & 11\end{array}$

4 Bessel case $\quad 13$

$\begin{array}{lll}4.1 & \text { One-point function } & 13\end{array}$

$\begin{array}{lll}4.2 & \text { Bessel kernel and spectral form factor } & 16\end{array}$

$\begin{array}{lll}5 & \text { Low temperature expansion } & 17\end{array}$

6 Large $\nu$ regime $\quad 20$

6.1 Genus-zero part 21

6.2 Higher genus free energy and constitutive relation 24

$\begin{array}{lll}6.3 & \text { Volume of } \mathrm{U}(\nu) & 29\end{array}$

$\begin{array}{ll}6.4 \text { Correlators at large } \nu & 29\end{array}$

7 Conclusions and outlook $\quad 32$

\section{Introduction}

Jackiw-Teitelboim (JT) gravity [1, 2] is a simple model of 2d gravity coupled to a scalar field, which serves as a solvable example of the $\mathrm{AdS}_{2} / \mathrm{CFT}_{1}$ correspondence [3-6]. In a recent paper [7], Saad, Shenker, and Stanford showed that the sum over topologies in JT gravity is reproduced from a matrix model, based on the observation of Eynard and Orantin [8] that the recursion relation for the Weil-Petersson volume found by Mirzakhani [9] can be recast as a topological recursion for a certain double-scaled matrix model [10]. This clearly shows that JT gravity on asymptotically $\mathrm{AdS}_{2}$ is holographically dual to an ensemble of one-dimensional quantum systems, where the Hamiltonian of a quantum system plays the role of a random matrix.

In our previous papers [11, 12], by generalizing the method of Zograf [13], we have developed a systematic technique of computing the genus expansion of the connected correlators $\left\langle Z\left(\beta_{1}\right) \cdots Z\left(\beta_{n}\right)\right\rangle_{c}$ of the thermal partition functions $Z\left(\beta_{i}\right)=\operatorname{Tr} e^{-\beta_{i} H}$ in the JT gravity matrix model. In particular, we found that the JT gravity matrix model is nothing 
but a special case of the Kontsevich-Witten (KW) topological gravity [14, 15] where infinite number of couplings are turned on with a specific value.

In this paper, we will show that a similar story holds for JT supergravity as well. In a paper by Stanford and Witten [16], it was shown that the matrix model description in [7] can be generalized to JT supergravity and classified by the Altland-Zirnbauer ensemble depending on the symmetry of the system. In this paper we will focus on the case of JT supergravity on orientable surfaces without time-reversal symmetry. As discussed in [16-18], in this case the corresponding matrix model computes the volume of the moduli space of super Riemann surfaces and it is characterized by the Brezin-Gross-Witten (BGW) $\tau$-function of the KdV hierarchy. We find that the matrix model of JT supergravity is a special case of the BGW model with infinite number of couplings turned on in a specific way, which is almost parallel to the case of bosonic JT gravity by replacing the KW model by the BGW model.

We first compute the genus expansion of JT supergravity using the cut-and-join representation of the BGW $\tau$-function [19] and study the low temperature behavior of the one-point function $\langle Z(\beta)\rangle$. Next, we consider the regime in which the Ramond-Ramond (RR) flux $\nu$ is large:

$$
\nu \gg 1, \quad \hbar \ll 1 \text { with } q=\hbar \nu \text { : fixed, }
$$

where $\hbar$ is the genus-counting parameter. In this regime we show that the higher genus free energy of the BGW model is written in terms of the genus-zero quantities. This kind of relation is known as the constitutive relation $[20,21]$. This enables us to study the genus expansion of connected correlators $\left\langle Z\left(\beta_{1}\right) \cdots Z\left(\beta_{n}\right)\right\rangle_{c}$ by the standard technique of the old matrix model (see [22] for a review).

This paper is organized as follows. In section 2, we show that the matrix model of JT supergravity is nothing but the BGW model with infinite number of couplings turned on in a specific way. In section 3, we compute the genus expansion of the BGW free energy using the cut-and-join operator. We also summarize the known result of the BGW $\tau$ function and study numerically the large genus behavior of the volume of the moduli space of super Riemann surfaces. In section 4, we consider the Bessel case where all couplings are set to zero in the BGW model. We propose a non-perturbative completion of the genus expansion of the one-point function $\langle Z(\beta)\rangle$ in the Bessel case. In section 5, we study the low temperature behavior of the one-point function $\langle Z(\beta)\rangle$ in JT supergravity and find the all-genus result at the lower orders in the low temperature expansion. In section 6 , we consider JT supergravity in the large $\nu$ regime (1.1) and derive the constitutive relation for the free energy. We also find that there are additional $q$-dependent constants in the free energy coming from the volume of the unitary group $\mathrm{U}(\nu)$. Finally in section 7 we conclude with some future directions.

\section{JT supergravity and Brezin-Gross-Witten $\tau$-function}

In this section we will show that the connected $n$-point function $\left\langle Z\left(\beta_{1}\right) \cdots Z\left(\beta_{n}\right)\right\rangle_{c}$ in JT supergravity is written in terms of the Brezin-Gross-Witten (BGW) $\tau$-function in a specific background. 
Let us first recall the result in [16]. As explained in [16], the expectation value in the supergravity normalization $\langle Z(\beta)\rangle_{\mathrm{SJT}}$ and that in the matrix model normalization $\langle Z(\beta)\rangle_{\text {mat }}$ are related by

$$
\langle Z(\beta)\rangle_{\mathrm{SJT}}=\nu+2\langle Z(\beta)\rangle_{\mathrm{mat}},
$$

where $\nu$ denotes the RR flux. The first term $\nu$ on the right hand side of $(2.1)$ counts the number of supersymmetric ground states, while the factor of 2 in front of $\langle Z(\beta)\rangle_{\text {mat }}$ comes from the two-fold degeneracy of the spectrum. In this paper, we will use the matrix model normalization unless otherwise stated. From the matrix model picture, the non-zero $\nu$ corresponds to the ensemble with $\mathrm{U}(N+\nu) \times \mathrm{U}(N)$ symmetry. In the Altland-Zirnbauer classification [23] it corresponds to the $(\boldsymbol{\alpha}, \boldsymbol{\beta})=(1+2 \nu, 2)$ case. In this ensemble, the supercharge takes the form

$$
Q=\left(\begin{array}{cc}
0 & M \\
M^{\dagger} & 0
\end{array}\right)
$$

where $M$ is an $(N+\nu) \times N$ complex matrix.

The correlator $\left\langle Z\left(\beta_{1}\right) \cdots Z\left(\beta_{n}\right)\right\rangle_{c}$ in JT supergravity is constructed by gluing several pieces of building blocks: the disk and the trumpet partition functions of the super Schwarzian mode, and the Weil-Petersson volume of the moduli space of super Riemann surfaces. The super Schwarzian partition functions on the disk and the trumpet are computed in [16]

$$
\begin{gathered}
Z^{\text {disk }}(\beta, b)=\frac{1}{2} Z_{\mathrm{SJT}}^{\text {disk }}(\beta)=e^{S_{0}} \frac{e^{\frac{\pi^{2}}{\beta}}}{\sqrt{2 \pi \beta}}, \\
Z^{\text {trumpet }}(\beta, b)=\frac{1}{2} Z_{\mathrm{SJT}}^{\text {trumpet }}(\beta, b)=\frac{1}{2} \frac{e^{-\frac{b^{2}}{4 \beta}}}{\sqrt{2 \pi \beta}},
\end{gathered}
$$

where we have used the matrix model normalization. Here and in the rest of this section we set $\nu=0$ for simplicity.

The volume of super moduli space is studied in [17]. The convention of [17] is different from that in [16] and we follow the definition in [16] in which the volume is given by

$$
\begin{aligned}
V_{g, n}\left(b_{1}, \cdots, b_{n}\right) & =(-1)^{n} 2^{1-g} \int_{\overline{\mathcal{M}}_{g, n}} \Theta_{g, n} \exp \left(2 \pi^{2} \kappa+\sum_{i=1}^{n} \frac{b_{i}^{2}}{2} \psi_{i}\right) \\
& =(-1)^{n} \int_{\overline{\mathcal{M}}_{g, n}} \Theta_{g, n} \exp \left(\pi^{2} \kappa+\sum_{i=1}^{n} \frac{b_{i}^{2}}{4} \psi_{i}\right) .
\end{aligned}
$$

Here $\kappa$ is the first Miller-Morita-Mumford class, $\psi_{i}$ is the first Chern class of the line bundle whose fiber is the cotangent space to the marked point $p_{i}$ on the Riemann surface, $\Theta_{g, n} \in H^{4 g-4+2 n}\left(\overline{\mathcal{M}}_{g, n}\right)$ is the $\Theta$-class introduced by Norbury in [24], and $b_{i}$ are the lengths of the geodesic boundaries. The geometric meaning of the $\Theta$-class is explained in [18]. When going from the first line to the second line of (2.4) we have used the selection rule

$$
\int_{\overline{\mathcal{M}}_{g, n}} \Theta_{g, n} \kappa^{\ell} \prod_{i} \psi_{i}^{k_{i}}=0 \quad \text { unless } \quad \ell+\sum_{i} k_{i}=g-1 .
$$


The sign $(-1)^{n}$ in (2.4) is important as we will see in later sections. Our definition (2.4) differs from that in [17] by a factor of $2^{-n}$.

Combining the trumpet partition functions and the Weil-Petersson volume of super moduli space, the $n$-point function for $n \geq 3$ is written as

$$
\left\langle Z\left(\beta_{1}\right) \cdots Z\left(\beta_{n}\right)\right\rangle_{c}=\sum_{g=0}^{\infty} e^{-S_{0}(2 g-2+n)} \int_{0}^{\infty} \prod_{i=1}^{n} b_{i} d b_{i} Z^{\text {trumpet }}\left(\beta_{i}, b_{i}\right) V_{g, n}\left(b_{1}, \cdots, b_{n}\right) .
$$

To simplify the expression, it is convenient to rescale $\beta$ by a factor of $\pi^{2}$

$$
\beta \rightarrow \pi^{2} \beta
$$

Then using the selection rule (2.5) we find

$$
\left\langle Z\left(\beta_{1}\right) \cdots Z\left(\beta_{n}\right)\right\rangle_{c}=\sum_{g=0}^{\infty} \hbar^{2 g-2+n} \prod_{i=1}^{n}\left(\frac{\beta_{i}}{2 \pi}\right)^{\frac{1}{2}} \int_{\overline{\mathcal{M}}_{g, n}} \Theta_{g, n} \frac{e^{\kappa}}{\prod_{i=1}^{n}\left(\beta_{i} \psi_{i}-1\right)} .
$$

Here we have introduced the genus-counting parameter $\hbar$ as

$$
\hbar=\pi e^{-S_{0}} .
$$

For the one- and two-point functions, we need to treat the genus-zero contribution separately. In the convention (2.7) and (2.9), the genus-zero part of the one-point function, i.e. the disk partition function in $(2.3)$ is written as

$$
\langle Z(\beta)\rangle^{g=0}=\frac{e^{\frac{1}{\beta}}}{\sqrt{2 \pi \beta \hbar}} .
$$

The expression (2.8) is similar to the bosonic case studied in [11, 12]. In the bosonic JT gravity, the $n$-point correlator is obtained by acting the boundary creation operator to the free energy of Kontsevich-Witten (KW) topological gravity. As emphasized in [11], the bosonic JT gravity corresponds to a background of topological gravity where the infinite number of couplings are turned on with a specific value

$$
t_{0}=t_{1}=0, \quad t_{n}=\frac{(-1)^{n}}{(n-1) !}(n \geq 2) .
$$

We will see that a similar relation holds for JT supergravity as well. In the case of JT supergravity, the $n$-point correlator is obtained by acting the boundary creation operator to the free energy of Brezin-Gross-Witten (BGW) model [25-28]. In fact, (2.8) is written as

$$
\left\langle Z\left(\beta_{1}\right) \cdots Z\left(\beta_{n}\right)\right\rangle_{c}=\left.B\left(\beta_{1}\right) \cdots B\left(\beta_{n}\right) G\left(1,\left\{t_{k}\right\}\right)\right|_{t_{k}=0}
$$

where we introduced the boundary creation operator $B(\beta)$

$$
B(\beta)=-\frac{\hbar}{\sqrt{2 \pi}} \sum_{k=0} \beta^{k+\frac{1}{2}} \partial_{k}
$$


with

$$
\partial_{k}=\frac{\partial}{\partial t_{k}}
$$

and the generating function of the intersection numbers $G\left(s,\left\{t_{k}\right\}\right)$

$$
G\left(s,\left\{t_{k}\right\}\right)=\sum_{g, n} \frac{\hbar^{2 g-2}}{n !} \sum_{\vec{k} \in \mathbb{N}^{n}} \int_{\overline{\mathcal{M}}_{g, n}} \Theta_{g, n} e^{s \kappa} \prod_{i=1}^{n} \psi_{i}^{k_{i}} t_{k_{i}} .
$$

As shown in [17], the $\kappa$-class can be replaced by some combination of $\psi$-classes and the result of this manipulation is summarized as ${ }^{1}$

$$
G\left(s,\left\{t_{k}\right\}\right)=F\left(\left\{t_{k}+\gamma_{k} s^{k}\right\}\right),
$$

where $F\left(\left\{t_{k}\right\}\right)$ is given by

$$
F\left(\left\{t_{k}\right\}\right)=\sum_{g, n} \frac{\hbar^{2 g-2}}{n !} \sum_{\vec{k} \in \mathbb{N}^{n}} \int_{\overline{\mathcal{M}}_{g, n}} \Theta_{g, n} \prod_{i=1}^{n} \psi_{i}^{k_{i}} t_{k_{i}},
$$

and $\gamma_{k}$ in (2.16) is given by

$$
\gamma_{0}=0, \quad \gamma_{k}=\frac{(-1)^{k-1}}{k !} \quad(k \geq 1) .
$$

Finally we arrive at our master equation

$$
\left\langle Z\left(\beta_{1}\right) \cdots Z\left(\beta_{n}\right)\right\rangle_{c}=\left.B\left(\beta_{1}\right) \cdots B\left(\beta_{n}\right) F\left(\left\{t_{k}\right\}\right)\right|_{t_{k}=\gamma_{k}} .
$$

In [17] it is proved that $F\left(\left\{t_{k}\right\}\right)$ in (2.17) is nothing but the free energy of the BGW unitary matrix model [25-28]

$$
e^{F}=\lim _{N \rightarrow \infty} \int_{\mathrm{U}(N)} d U e^{\frac{1}{\hbar} \operatorname{Tr}\left(A^{\dagger} U+A U^{\dagger}\right),}
$$

and the coupling $t_{k}$ is given by the Miwa transform

$$
t_{k}=\frac{1}{2 k+1} \operatorname{Tr}\left(A^{\dagger} A\right)^{-k-\frac{1}{2}} .
$$

Thus we find that the correlator in JT supergravity is computed from the BGW model with a specific background $t_{k}=\gamma_{k}$ with (2.18). This is analogous to the situation in the bosonic JT gravity as mentioned above.

Note that the disk partition function (2.10) is written as

$$
\langle Z(\beta)\rangle^{g=0}=\int_{0}^{\infty} d E \rho_{0}(E) e^{-\beta E},
$$

where the genus-zero eigenvalue density $\rho_{0}(E)$ is given by

$$
\rho_{0}(E)=\frac{\cosh 2 \sqrt{E}}{\sqrt{2 E} \pi \hbar} .
$$

\footnotetext{
${ }^{1} \mathrm{~A}$ similar relation for the bosonic case was obtained in [29, 30].
} 
From this eigenvalue density $\rho_{0}(E)$ one can read off the spectral curve [16]

$$
y=-\frac{\cos (2 z)}{\sqrt{2} z}
$$

with $E=-z^{2}$. From the data of spectral curve, one can compute the genus expansion of free energy systematically by using the Eynard-Orantin's topological recursion [10]. Alternatively, there is a more direct method to compute the genus expansion, known as the cut-and-join operator which we will review in the next section.

\section{Free energy of Brezin-Gross-Witten model}

\subsection{Cut-and-join operator}

As shown in [19] the free energy of BGW model is systematically computed by the so-called cut-and-join operator $M$

$$
e^{F\left(p_{i}, \hbar\right)}=e^{\hbar M} \cdot 1
$$

where $M$ is given by $[19,31]$

$$
M=\frac{1-4 \nu^{2}}{8} p_{1}+\frac{1}{2} \sum_{i, j \in 2 \mathbb{N}+1} i j p_{i+j+1} \frac{\partial^{2}}{\partial p_{i} \partial p_{j}}+\sum_{i, j \in 2 \mathbb{N}+1}(i+j-1) p_{i} p_{j} \frac{\partial}{\partial p_{i+j-1}} .
$$

The sum of $i, j$ run over positive odd integers. $p_{2 k+1}$ and the coupling $t_{k}$ in the previous section are related by

$$
p_{2 k+1}=\frac{t_{k}}{\hbar(2 k-1) ! !}
$$

Let us briefly recall the derivation of (3.1). As we review in section 3.3, the BGW $\tau$-function $\tau\left(p_{i}, \hbar\right)=e^{F\left(p_{i}, \hbar\right)}$ obeys the Virasoro constraint $\mathcal{L}_{m} \tau\left(p_{i}, \hbar\right)=0$ where $\mathcal{L}_{m}$ is given by (3.35). Let us consider the following linear combination of the Virasoro constraint

$$
\sum_{m=0}^{\infty} p_{2 m+1} \mathcal{L}_{m} \tau\left(p_{i}, \hbar\right)=0
$$

One can show that this is rewritten as [19]

$$
M \tau\left(p_{i}, \hbar\right)=\frac{1}{\hbar} D \tau\left(p_{i}, \hbar\right),
$$

where $M$ is the cut-and-join operator in (3.2) and $D$ is the Euler operator

$$
D=\sum_{i \in 2 \mathbb{N}+1} i p_{i} \frac{\partial}{\partial p_{i}}
$$

Due to the selection rule (2.5), $\tau=e^{F}$ defined by (2.17) has the property $\tau\left(p_{i}, \hbar\right)=$ $\tau\left(\hbar^{i} p_{i}, 1\right)$. This means that if we expand $\tau$ as

$$
\tau\left(p_{i}, \hbar\right)=1+\sum_{k=1}^{\infty} \hbar^{k} \tau^{(k)}\left(p_{i}\right)
$$


the coefficients satisfy

$$
D \tau^{(k)}\left(p_{i}\right)=k \tau^{(k)}\left(p_{i}\right)
$$

Therefore using (3.5) we find

$$
M \tau^{(k)}\left(p_{i}\right)=(k+1) \tau^{(k+1)}\left(p_{i}\right) \Rightarrow \tau^{(k)}\left(p_{i}\right)=\frac{M^{k}}{k !} \cdot 1,
$$

which implies our desired relation (3.1).

For $\nu=0$, one can compute the first few terms of free energy by expanding (3.1)

$$
F=\frac{1}{8} p_{1} \hbar+\frac{1}{16} p_{1}^{2} \hbar^{2}+\left(\frac{3}{128} p_{3}+\frac{1}{24} p_{1}^{3}\right) \hbar^{3}+\left(\frac{9}{128} p_{3} p_{1}+\frac{1}{32} p_{1}^{4}\right) \hbar^{4}+\cdots .
$$

Then, from (3.3) the genus expansion of free energy is obtained as

$$
\sum_{g=0}^{\infty} \hbar^{2 g-2} F_{g}\left(\left\{t_{k}\right\}\right)=F\left(p_{2 k+1}=\frac{t_{k}}{\hbar(2 k-1) ! !}, \hbar\right) .
$$

Using the dictionary (3.11) the free energy for $\nu=0$ is obtained from (3.10) as

$$
\begin{aligned}
& F_{0}=0 \\
& F_{1}=-\frac{1}{8} \log \left(1-t_{0}\right) \\
& F_{2}=\frac{3 t_{1}}{128\left(1-t_{0}\right)^{3}}, \\
& F_{3}=\frac{15 t_{2}}{1024\left(1-t_{0}\right)^{5}}+\frac{63 t_{1}^{2}}{1024\left(1-t_{0}\right)^{6}}, \\
& F_{4}=\frac{2407 t_{1}^{3}}{4096\left(1-t_{0}\right)^{9}}+\frac{8625 t_{1} t_{2}}{32768\left(1-t_{0}\right)^{8}}+\frac{525 t_{3}}{32768\left(1-t_{0}\right)^{7}} .
\end{aligned}
$$

Notice that the genus-zero free energy vanishes identically in the BGW model. This is quite different from the KW case. In particular, we cannot use the KdV equation to solve the higher genus free energy recursively staring from the genus-zero potential $u_{0}=\partial_{0}^{2} F_{0}$, which was heavily utilized in the Zograf's approach [13]. As we will see in section 6 this problem is avoided in the large $\nu$ regime (1.1). Note also that the $t_{0}$-dependence is recovered from the $t_{0}=0$ result by the following rescaling

$$
F_{g} \rightarrow \frac{F_{g}}{\left(1-t_{0}\right)^{2 g-2}}, \quad t_{n} \rightarrow \frac{t_{n}}{1-t_{0}} \quad(n \geq 1) .
$$

For non-zero $\nu$, the $\nu$-dependence appears through the combination [19]

$$
B_{k}=\prod_{i=1}^{k}\left[(2 i-1)^{2}-4 \nu^{2}\right]
$$

For instance, $F_{1}$ is given by

$$
F_{1}=-\frac{B_{1}}{8} \log \left(1-t_{0}\right)
$$


The higher genus corrections are easily obtained from the cut-and-join operator

$$
\begin{aligned}
& F_{2}=\frac{t_{1}}{384} B_{2}, \\
& F_{3}=-\frac{1}{768} t_{1}^{2} B_{2}+\frac{5 t_{1}^{2}+t_{2}}{15360} B_{3}, \\
& F_{4}=\frac{t_{1}^{3}}{1152} B_{2}-\frac{13 t_{1}^{3}+3 t_{1} t_{2}}{9216} B_{3}+\frac{56 t_{1}^{3}+21 t_{1} t_{2}+t_{3}}{688128} B_{4} .
\end{aligned}
$$

Here we have set $t_{0}=0$ for simplicity. The $t_{0}$-dependence is recovered from the replacement (3.13).

\subsection{String equation}

One can also compute the genus expansion using the string equation for the complex matrix model [32-35] which reads ${ }^{2}$

$$
\hbar^{2} \nu^{2}=\frac{\hbar^{2}}{4}\left[\left(\partial_{0} \mathcal{R}\right)^{2}-2 \mathcal{R} \partial_{0}^{2} \mathcal{R}\right]-2 u \mathcal{R}^{2},
$$

where $\mathcal{R}$ is a combination of the Gelfand-Dikii differential polynomial $\mathcal{R}_{k}$

$$
\mathcal{R}=\sum_{k=0}^{\infty} \widetilde{t}_{k} \mathcal{R}_{k},
$$

with

$$
\widetilde{t}_{k}=t_{k}-\delta_{k, 0}
$$

Note that $\mathcal{R}_{k}$ is normalized as

$$
\mathcal{R}_{0}=1, \quad \mathcal{R}_{1}=u, \quad \mathcal{R}_{2}=\frac{u^{2}}{2}+\frac{\hbar^{2} \partial_{0}^{2} u}{12}, \quad \mathcal{R}_{k}=\frac{u^{k}}{k !}+\cdots,
$$

where $u$ is defined by

$$
u=\hbar^{2} \partial_{0}^{2} F
$$

$\mathcal{R}_{k}$ can be computed recursively as

$$
(2 k+1) \partial_{0} \mathcal{R}_{k+1}=\frac{\hbar^{2}}{4} \partial_{0}^{3} \mathcal{R}_{k}+2 u \partial_{0} \mathcal{R}_{k}+\left(\partial_{0} u\right) \mathcal{R}_{k},
$$

and $u$ obeys the KdV flow equation

$$
\partial_{k} u=\partial_{0} \mathcal{R}_{k+1}
$$

As discussed in [35], the string equation (3.17) gives a non-perturbative definition of the free energy of type 0A minimal superstring theory. When the $k$-th coupling is turned

\footnotetext{
${ }^{2}$ The role of the string equation (3.17) in the context of JT supergravity has been discussed recently in $[36-38]$.
} 
on $t_{k} \neq 0$ with higher couplings set to zero $t_{n}=0(n \geq k+1)$, the string equation (3.17) describes the $(2,4 k)$ minimal superstring theory. In this case, the differential equation (3.17) is solved with the boundary condition [35]

$$
u \sim \begin{cases}0, & \left(t_{0} \rightarrow \infty\right), \\ \left(1-t_{0}\right)^{\frac{1}{k}}, & \left(t_{0} \rightarrow-\infty\right) .\end{cases}
$$

In the present case of JT supergravity with the infinite number of couplings $t_{n}=\gamma_{n}$ turned on, it is not clear what is the appropriate boundary condition for $u$ to solve the string equation (3.17).

However, we are only interested in the formal solution of (3.17) in the small $\hbar$ expansion $u=\sum_{g=0}^{\infty} \hbar^{2 g} u_{g}$. For this purpose, it is not necessary to know the global analytic behavior of $u$ as a function of $t_{0}$. It turns out that we can solve the string equation (3.17) order by order in the $\hbar$ expansion just by algebraic manipulations since $u_{g}$ is a polynomial in $\left(1-t_{0}\right)^{-1}$. Let us see this for the first few orders. At the order $\mathcal{O}\left(\hbar^{0}\right)$, the string equation (3.17) implies $u_{0}=0$. At the next order $\mathcal{O}\left(\hbar^{2}\right)$, noticing the relation

$$
\left.\mathcal{R}\right|_{u_{0}=0}=t_{0}-1+\mathcal{O}\left(\hbar^{2}\right),
$$

and by equating the order $\mathcal{O}\left(\hbar^{2}\right)$ terms on the both sides of $(3.17)$, we find

$$
\nu^{2}=\frac{1}{4}-2 u_{1}\left(t_{0}-1\right)^{2} .
$$

From the relation $u_{1}=\partial_{0}^{2} F_{1}$, this reproduces the genus-one free energy $F_{1}$ in (3.15). In this manner we can compute the higher genus free energy $F_{g}$ by solving the string equation (3.17) order by order in the $\hbar$ expansion. We have checked that the free energy obtained from (3.17) agrees with that computed from the cut-and-join operator in the previous subsection.

\subsection{Free fermion representation of BGW $\tau$-function}

The BGW $\tau$-function satisfies the KdV hierarchy and hence it admits the free fermion representation

$$
e^{F}=\langle t \mid V\rangle .
$$

Here $\langle t|$ denotes the coherent state of free boson $\left[\alpha_{n}, \alpha_{m}\right]=n \delta_{n+m, 0}$

$$
\langle t|=\langle 0| \exp \left(\sum_{n \geq 0} \frac{t_{n}}{\hbar(2 n+1) ! !} \alpha_{2 n+1}\right),
$$

and $|V\rangle$ is expanded in terms of the free fermion obeying $\left\{\psi_{r}, \psi_{s}^{*}\right\}=\delta_{r+s, 0}$

$$
|V\rangle=\exp \left(\sum_{m, n \geq 0} A_{m, n} \psi_{-m-\frac{1}{2}} \psi_{-n-\frac{1}{2}}^{*}\right)|0\rangle .
$$


Note that the boson $\alpha_{n}$ and the fermion $\psi_{r}$ are related by the usual bosonization

$$
\alpha_{n}=\sum_{r \in \mathbb{Z}+\frac{1}{2}}: \psi_{r} \psi_{n-r}^{*}:
$$

For the BGW model, the coefficient $A_{m, n}$ is explicitly given by [39]

$$
\begin{aligned}
& A_{m, n}=(-1)^{n}\left(\frac{\hbar}{2}\right)^{m+n+1} \sum_{\substack{r \geq m+1, s \geq 0 \\
r+s=m+n+1}} \frac{r-s}{r+s} \frac{a_{r}(\nu) a_{s}(\nu)}{r ! s !}, \\
& a_{r}(\nu)=\left(\frac{1}{2}+\nu\right)_{r}\left(\frac{1}{2}-\nu\right)_{r},
\end{aligned}
$$

where $(x)_{n}=x(x+1) \cdots(x+n-1)$ is the Pochhammer symbol. The generating function of $A_{m, n}$ is given by the modified Bessel functions $I_{\nu}(z), K_{\nu}(z)$ [39]

$$
\begin{aligned}
& \left.\sum_{m, n \geq 0} A_{m, n} z^{-m-1} w^{-n-1}\right|_{\hbar=1} \\
& =\frac{2 e^{z-w} \sqrt{4 \pi^{2} z w}}{z^{2}-w^{2}}\left(K_{\nu}(z) w I_{\nu}^{\prime}(w)+I_{\nu}(w) z K_{\nu}^{\prime}(z)\right)-\frac{1}{z-w},
\end{aligned}
$$

where we set $\hbar=1$ for simplicity. The $\hbar$-dependence can be easily recovered by multiplying $A_{m, n}$ by $\hbar^{m+n+1}$.

The important property of the state $|V\rangle$ is that it satisfies the Virasoro constraint $[34,40-42]$

$$
\mathcal{L}_{m}|V\rangle=0 \quad(m \geq 0),
$$

where the Virasoro generator $\mathcal{L}_{m}$ is given by

$$
\begin{aligned}
\mathcal{L}_{m} & =-\frac{\alpha_{2 m+1}}{2 \hbar}+L_{m}, \\
L_{m} & =\frac{1}{4} \sum_{n \in \mathbb{Z}}: \alpha_{2 m+2 n+1} \alpha_{-2 n-1}:+\frac{1-4 \nu^{2}}{16} \delta_{m, 0} .
\end{aligned}
$$

In terms of the couplings $\left\{t_{n}\right\}$ the Virasoro generator is written as

$$
\mathcal{L}_{m}=\frac{\hbar^{2}}{4} \sum_{i+j=m-1}(2 i+1) ! !(2 j+1) ! ! \partial_{i} \partial_{j}+\frac{1}{2} \sum_{i \geq 0} \frac{(2 i+2 m+1) ! !}{(2 i-1) ! !} \widetilde{t}_{i} \partial_{i+m}+\frac{1-4 \nu^{2}}{16} \delta_{m, 0} .
$$

One can check the Virasoro constraint (3.33) order by order in the $\hbar$-expansion. To see this let us expand $|V\rangle$ as

$$
|V\rangle=\sum_{n=0}^{\infty} \hbar^{n}\left|V_{n}\right\rangle
$$

From the explicit form of $A_{m, n}$ in (3.31), we find the first few terms of $\left|V_{n}\right\rangle$

$$
\begin{aligned}
\left|V_{0}\right\rangle & =|0\rangle \\
\left|V_{1}\right\rangle & =\frac{1}{2} a_{1} \psi_{-\frac{1}{2}} \psi_{-\frac{1}{2}}^{*}|0\rangle, \\
\left|V_{2}\right\rangle & =\frac{1}{8} a_{2}\left(\psi_{-\frac{3}{2}} \psi_{-\frac{1}{2}}^{*}-\psi_{-\frac{1}{2}} \psi_{-\frac{3}{2}}^{*}\right)|0\rangle \\
\left|V_{3}\right\rangle & =\frac{1}{48}\left[a_{3}\left(\psi_{-\frac{5}{2}} \psi_{-\frac{1}{2}}^{*}+\psi_{-\frac{1}{2}} \psi_{-\frac{5}{2}}^{*}\right)-\left(a_{3}+a_{2} a_{1}\right) \psi_{-\frac{3}{2}} \psi_{-\frac{3}{2}}^{*}\right]|0\rangle .
\end{aligned}
$$


Using the above expressions we have checked the Virasoro constraint at order $\mathcal{O}\left(\hbar^{n}\right)(0 \leq$ $n \leq 2)$

$$
L_{m}\left|V_{n}\right\rangle-\frac{1}{2} \alpha_{2 m+1}\left|V_{n+1}\right\rangle=0 .
$$

As an application of this formalism, let us show that the genus-zero one-point function $\langle Z(\beta)\rangle^{g=0}$ is independent of the RR flux $\nu$. As shown in [12], the one-point function $\langle Z(\beta)\rangle$ is written as

$$
\langle Z(\beta)\rangle=\frac{\langle t|\widehat{Z}(\beta)| V\rangle}{\langle t \mid V\rangle}
$$

where $\widehat{Z}(\beta)$ is given by

$$
\widehat{Z}(\beta)=-\frac{1}{\sqrt{2 \pi}} \sum_{n \in \mathbb{Z}} \beta^{n+\frac{1}{2}} \frac{\alpha_{2 n+1}}{(2 n+1) ! !} .
$$

At genus-zero $|V\rangle$ can be replaced by $\left|V_{0}\right\rangle=|0\rangle$

$$
\langle Z(\beta)\rangle^{g=0}=\frac{\langle t|\widehat{Z}(\beta)| 0\rangle}{\langle t \mid 0\rangle}
$$

which shows that $\langle Z(\beta)\rangle^{g=0}$ is independent of $\nu$. Thus we can use the same expression (2.10) for the disk partition function for the non-zero $\nu$ case as well. Similarly, the genus-zero part of the two-point function is independent of $\nu$

$$
\left\langle Z\left(\beta_{1}\right) Z\left(\beta_{2}\right)\right\rangle_{c}^{g=0}=\left\langle 0\left|\widehat{Z}\left(\beta_{1}\right) \widehat{Z}\left(\beta_{1}\right)\right| 0\right\rangle=\frac{\sqrt{\beta_{1} \beta_{2}}}{2 \pi\left(\beta_{1}+\beta_{2}\right)} .
$$

\subsection{Large genus asymptotics}

It is interesting to consider the large genus asymptotics of the Weil-Petersson volume $V_{g, 1}(b)$ of super Riemann surface, from which we can extract a possible non-perturbative effects in the $\hbar$-expansion. As usual in string perturbation theory, $V_{g, 1}(b)$ grows as $(2 g)$ ! and the genus expansion is an asymptotic series. Note that for the bosonic case $V_{g, 1}(b)$ behaves in the large $g$ limit as $[7,13,43]$

$$
V_{g, 1}(b) \sim \frac{2}{(2 \pi)^{\frac{9}{2}}}\left(4 \pi^{2}\right)^{2 g} \Gamma(2 g-3 / 2) \frac{2}{b} \sinh \frac{b}{2},
$$

while in the super case $V_{g, 1}(b)$ behaves as [16]

$$
V_{g, 1}(b) \sim-\frac{2^{3}}{(2 \pi)^{\frac{7}{2}}}\left(\frac{\pi}{\sqrt{2}}\right)^{2 g} \Gamma(2 g-3 / 2) \frac{4}{b} \sinh \frac{b}{4} .
$$

In this section we will check (3.44) and study its higher order corrections by means of the numerical analysis using the Richardson transformation.

Once we know the genus- $g$ free energy $F_{g}$ of the BGW model, the intersection number is computed as

$$
\int_{\overline{\mathcal{M}}_{g, 1}} \Theta_{g, 1} e^{\kappa} \psi_{1}^{d}=\left.\partial_{d} F_{g}\right|_{t_{n}=\gamma_{n}},
$$


and $V_{g, 1}(b)$ is given by

$$
\begin{aligned}
V_{g, 1}(b) & =-\int_{\overline{\mathcal{M}}_{g, 1}} \Theta_{g, 1} \exp \left(\pi^{2} \kappa+\frac{b^{2}}{4} \psi_{1}\right) \\
& =-\sum_{d=0}^{g-1} \pi^{2(g-1-d)}\left(\frac{b^{2}}{4}\right)^{d} \int_{\overline{\mathcal{M}}_{g, 1}} \Theta_{g, 1} e^{\kappa} \psi_{1}^{d} .
\end{aligned}
$$

We have computed $F_{g}$ up to $g=30$ using the cut-and-join operator. From these data, we can extract the large genus behavior of the intersection number (3.45) by the standard technique of the Richardson transformation. In order to find the asymptotic value of the series

$$
S_{g}=s_{0}+\frac{s_{1}}{g}+\frac{s_{2}}{g^{2}}+\cdots, \quad \lim _{g \rightarrow \infty} S_{g}=s_{0},
$$

it is convenient to construct its $k$-th Richardson transform

$$
S_{g}^{(k)}=\sum_{n=0}^{k} \frac{(-1)^{k+n}(g+n)^{n} S_{g+n}}{n !(k-n) !} .
$$

Then one can show that $S_{g}^{(k)}$ converges to $s_{0}$ much faster than the original series $S_{g}$

$$
S_{g}^{(k)}-s_{0}=\mathcal{O}\left(g^{-k-1}\right)
$$

Using this acceleration method, we find the asymptotic behavior of the intersection number

$$
\int_{\overline{\mathcal{M}}_{g, 1}} \Theta_{g, 1} e^{\kappa} \psi_{1}^{d} \sim \sqrt{\frac{2}{\pi^{3}}} 2^{-g} \frac{\Gamma(3 / 2)}{\Gamma(3 / 2+d)}\left(\frac{\pi}{4}\right)^{2 d} \sum_{n=0}^{\infty} f_{n}(d) \Gamma(2 g-3 / 2-n)
$$

where

$$
f_{0}=1, \quad f_{1}=-\frac{1}{8}-\frac{1}{\pi}+\frac{4 d-8 d^{2}}{\pi^{2}} .
$$

Let us check the behavior (3.50) numerically by the Richardson transformation. For instance, from (3.50) we expect that the combination

$$
A_{g}=\left.4 g^{2} \frac{\partial_{0} F_{g}}{\partial_{0} F_{g+1}}\right|_{t_{n}=\gamma_{n}}
$$

approaches a constant value at large genus

$$
\lim _{g \rightarrow \infty} A_{g}=2 .
$$

As we can see from figure 1 the Richardson transformation accelerates the convergence of $A_{g}$ to 2 . We have checked $f_{0}, f_{1}$ in (3.51) in a similar manner by constructing appropriate series which converge to $f_{0}, f_{1}$ at large genus (see e.g. [44] for the details of this procedure). 


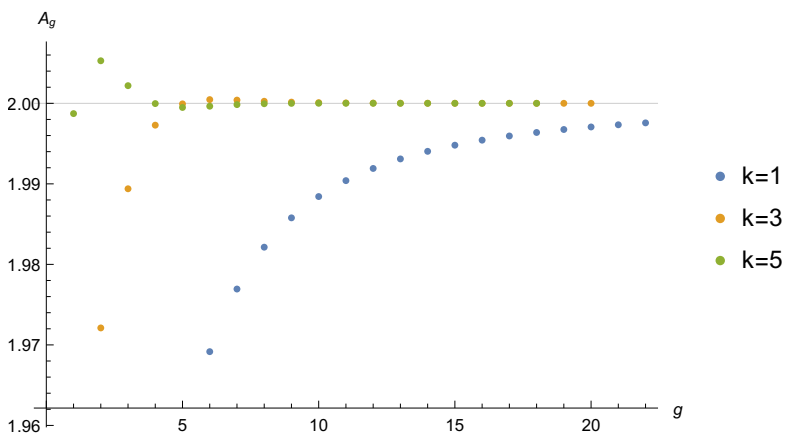

Figure 1. Plot of the $k$-th Richardson transform $A_{g}^{(k)}$ of $A_{g}$ in $(3.52)$ for $k=1,3,5$.

From (3.46) we can translate the asymptotic behavior of the intersection number (3.50) to that of the Weil-Petersson volume of super moduli space

$$
\begin{aligned}
V_{g, 1}(b) \sim & -\frac{2^{3}}{(2 \pi)^{\frac{7}{2}}}\left(\frac{\pi}{\sqrt{2}}\right)^{2 g}\left[\Gamma(2 g-3 / 2) \frac{4}{b} \sinh \frac{b}{4}\right. \\
& \left.+\Gamma(2 g-5 / 2)\left(-\frac{\sinh \left(\frac{b}{4}\right)}{2 b}-\frac{4 \sinh \left(\frac{b}{4}\right)}{b \pi}+\frac{8 b \cosh \left(\frac{b}{4}\right)-\left(32+b^{2}\right) \sinh \left(\frac{b}{4}\right)}{2 b \pi^{2}}\right)+\cdots\right] .
\end{aligned}
$$

The first line agrees with the result (3.44) found in [16], while the second line is our new prediction. Our result implies that there is a non-perturbative correction of the order

$$
e^{-\frac{\sqrt{2}}{\pi} e^{S_{0}}}=e^{-\frac{\sqrt{2}}{\hbar}}
$$

which is doubly exponential in $S_{0}$. It would be interesting to identify the physical origin of this D-brane like effect and clarify its meaning in JT supergravity.

\section{Bessel case}

If we ignore the $\kappa$-class, the BGW model reduces to the so-called Bessel case corresponding to the spectral curve $y=-\frac{1}{2 z}$, or the genus-zero eigenvalue density $\rho_{0}(E)=\frac{1}{\sqrt{2 E} \pi \hbar}$. This density has the behavior $\rho_{0}(E) \sim E^{-\frac{1}{2}}$ near $E=0$, known as the "hard edge" of the spectrum. In this section we will first summarize the known results in the Bessel case and then study the non-perturbative aspects of the one-point function as well as the spectral form factor obtained from the two-point function. ${ }^{3}$

\subsection{One-point function}

The Bessel case corresponds to the trivial background $t_{n}=0(n \geq 0)$ in the BGW model. However, it is useful to turn on $t_{0} \neq 0$ and set other couplings $t_{n}=0(n \geq 1)$. Then the

\footnotetext{
${ }^{3}$ Similar analyses of the Bessel case in the context of JT supergravity have been done earlier in [36-38], where nonperturbative definitions of JT gravity and JT supergravities with/without time-reversal symmetry are proposed and the connection of the Bessel kernel approach to the string equation (3.17) is also discussed.
} 
potential $u=\hbar^{2} \partial_{0}^{2} F$ is given by

$$
u(x)=\frac{\left(1-4 \nu^{2}\right) \hbar^{2}}{8 x^{2}},
$$

where we introduced

$$
x:=1-t_{0} .
$$

The Baker-Akhiezer (BA) function is defined by a solution of the Schrödinger equation

$$
\frac{\hbar^{2}}{2} \partial_{x}^{2} \psi+u(x) \psi=-E \psi .
$$

The solution satisfying the boundary condition $\psi(x=0)=0$ is given by the Bessel function

$$
\psi=\langle x \mid E\rangle=\frac{\sqrt{x}}{\hbar} J_{\nu}\left(\frac{x \sqrt{2 E}}{\hbar}\right) .
$$

This is normalized as

$$
\int_{0}^{\infty} d x\langle E \mid x\rangle\left\langle x \mid E^{\prime}\right\rangle=\delta\left(E-E^{\prime}\right)
$$

which is formally written as

$$
1=\int_{0}^{\infty} d x|x\rangle\langle x|
$$

It is also convenient to introduce the projector

$$
\Pi=\int_{0}^{1} d x|x\rangle\langle x|, \quad \Pi^{2}=\Pi .
$$

Then the eigenvalue density is given by [45]

$$
\rho(E)=\langle E|\Pi| E\rangle=\int_{0}^{1} d x\langle x \mid E\rangle^{2}=\frac{J_{\nu}(\xi)^{2}-J_{\nu-1}(\xi) J_{1+\nu}(\xi)}{2 \hbar^{2}},
$$

where

$$
\xi=\frac{\sqrt{2 E}}{\hbar} \text {. }
$$

Note that (4.8) is the all-genus result of the eigenvalue density. See figure 2 for the plot of $\rho(E)$ in (4.8) for $\nu=0$ and $\nu=10$. One can see that the genus-zero eigenvalue density $\rho_{0}(E) \sim E^{-\frac{1}{2}}$ appears as the envelope of the oscillating exact eigenvalue density $\rho(E)$.

Using this result, the exact partition function for the Bessel case is computed as

$$
\begin{aligned}
Z(\beta, \nu) & =\int_{0}^{\infty} d E \rho(E) e^{-\beta E} \\
& =\frac{(\sigma / 2)^{\nu+1}}{\Gamma(2+\nu)}{ }_{2} F_{2}\left(\nu+\frac{1}{2}, \nu+1 ; 2+\nu, 1+2 \nu ;-2 \sigma\right),
\end{aligned}
$$

where we introduced the parameter $\sigma$ by

$$
\sigma=\frac{1}{\hbar^{2} \beta}
$$




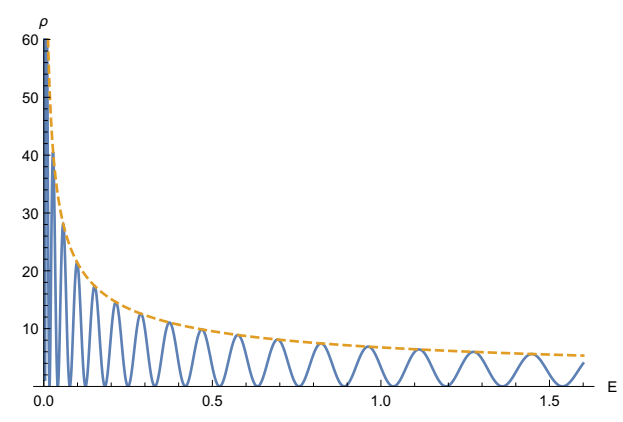

(a) $\nu=0$

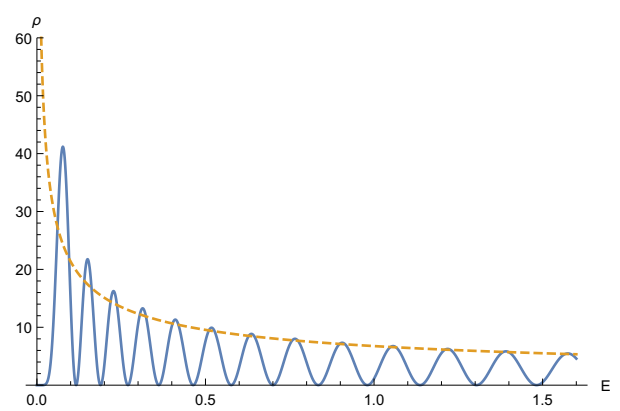

(b) $\nu=10$

Figure 2. Plot of the eigenvalue density of the Bessel case for (a) $\nu=0$ and (b) $\nu=10$ with $\hbar=1 / 30$. The solid curve is the plot of $\rho(E)$ in (4.8) while the dashed curve represents the genus-zero result $\rho_{0}(E)=\frac{1}{\sqrt{2 E} \pi \hbar}$.

One can check that this obeys a simple relation

$$
2 \partial_{\sigma} Z(\beta, \nu)=e^{-\sigma} I_{\nu}(\sigma)
$$

For integer $\nu$, we find

$$
\nu+2 Z(\beta, \nu)=e^{-\sigma}\left[a(\sigma, \nu) I_{\nu}(\sigma)+b(\sigma, \nu) I_{\nu-1}(\sigma)\right]
$$

where

$$
\begin{array}{ll}
a(\sigma, 0)=\sigma, & b(\sigma, 0)=\sigma, \\
a(\sigma, 1)=\sigma, & b(\sigma, 1)=\sigma+1, \\
a(\sigma, 2)=\sigma+2, & b(\sigma, 2)=\sigma+4+4 \sigma^{-1}, \\
a(\sigma, 3)=\sigma+6+6 \sigma^{-1}, & b(\sigma, 3)=\sigma+9+24 \sigma^{-1}+24 \sigma^{-2}, \\
a(\sigma, 4)=\sigma+12+32 \sigma^{-1}+32 \sigma^{-2}, & b(\sigma, 4)=\sigma+16+80 \sigma^{-1}+192 \sigma^{-2}+192 \sigma^{-3} .
\end{array}
$$

Interestingly, the relation (2.1) between the supergravity normalization and the matrix model normalization has a natural counterpart in the Bessel case (4.13) as well.

Let us consider the asymptotic genus expansion of the one-point function for $\nu=0$

$$
\begin{aligned}
Z(\beta, 0) & =\frac{1}{2} \sigma e^{-\sigma}\left[I_{0}(\sigma)+I_{1}(\sigma)\right] \\
& \simeq \frac{1}{\sqrt{2 \pi \beta \hbar}}\left(1-\frac{\hbar^{2} \beta}{8}-\frac{3 \hbar^{4} \beta^{2}}{128}-\cdots\right) \\
& =:-\frac{1}{\sqrt{2 \pi}} \sum_{g=0}^{\infty} c_{0}(g)\left(\hbar^{2} \beta\right)^{g-\frac{1}{2}}
\end{aligned}
$$

where $c_{0}(g)$ is given by

$$
c_{0}(g)=\frac{(2 g-1) ! !(2 g-3) ! !}{8^{g} g !} .
$$


It is interesting to notice that the coefficients of the genus expansion in (4.15) are negative. This is consistent with our expression of $\langle Z(\beta)\rangle$ in $(2.8)$ for $\kappa=0$

$$
Z(\beta, 0)=-\sqrt{\frac{\beta}{2 \pi}} \sum_{g \geq 0} \hbar^{2 g-1} \int_{\overline{\mathcal{M}}_{g, 1}} \frac{\Theta_{g, 1}}{1-\beta \psi_{1}} .
$$

Note that this minus sign comes from the definition (2.4) of the Weil-Petersson volume of the super Riemann surface. Nevertheless, the exact result in the first line of (4.15) is a positive function for $\beta>0$. A non-perturbative resummation of $c_{0}(g)$ is proposed in [46] in terms of the Tricomi's confluent hypergeometric function, which is different from our result (4.15). As we have seen above, the integral of the exact eigenvalue density (4.8) uniquely selects the modified Bessel function (4.15) as the non-perturbative completion. Note that this type of asymptotic expansion (4.15) has also appeared in the zero-dimensional Sine-Gordon model [47].

\subsection{Bessel kernel and spectral form factor}

The higher point functions in the Bessel case can in principle be obtained from a combination of the Darboux-Christoffel kernel

$$
K\left(E_{1}, E_{2}\right)=\left\langle E_{1}|\Pi| E_{2}\right\rangle=\int_{0}^{1} d x \psi\left(E_{1}\right) \psi\left(E_{2}\right) .
$$

Using the Schrödinger equation (4.3) one can show that

$$
\begin{aligned}
& \left(E_{1}-E_{2}\right) K\left(E_{1}, E_{2}\right) \\
& =\int_{0}^{1} d x\left[-\left(\frac{\hbar^{2}}{2} \partial_{x}^{2} \psi\left(E_{1}\right)+u \psi\left(E_{1}\right)\right) \psi\left(E_{2}\right)+\psi\left(E_{1}\right)\left(\frac{\hbar^{2}}{2} \partial_{x}^{2} \psi\left(E_{2}\right)+u \psi\left(E_{2}\right)\right)\right] \\
& =\int_{0}^{1} d x \frac{\hbar^{2}}{2} \partial_{x}\left[\psi\left(E_{1}\right) \partial_{x} \psi\left(E_{2}\right)-\psi\left(E_{2}\right) \partial_{x} \psi\left(E_{1}\right)\right] \\
& =\left.\frac{\hbar^{2}}{2}\left[\psi\left(E_{1}\right) \partial_{x} \psi\left(E_{2}\right)-\psi\left(E_{2}\right) \partial_{x} \psi\left(E_{1}\right)\right]\right|_{x=1} .
\end{aligned}
$$

Using the explicit form of the BA function (4.4) we find

$$
K\left(E_{1}, E_{2}\right)=\frac{J_{\nu}\left(\xi_{1}\right) \xi_{2} J_{\nu}^{\prime}\left(\xi_{2}\right)-J_{\nu}\left(\xi_{2}\right) \xi_{1} J_{\nu}^{\prime}\left(\xi_{1}\right)}{\hbar^{2}\left(\xi_{1}^{2}-\xi_{2}^{2}\right)}
$$

where $\xi$ is defined in (4.9). This is known as the Bessel kernel. The eigenvalue density is given by the diagonal part of the Darboux-Christoffel kernel

$$
\rho(E)=K(E, E)
$$

Now let us consider the two-point function in the Bessel case. The general expression of the two-point function is obtained in [48]

$$
\left\langle Z\left(\beta_{1}\right) Z\left(\beta_{2}\right)\right\rangle_{c}=\operatorname{Tr}\left[e^{-\beta_{1} H}(1-\Pi) e^{-\beta_{2} H} \Pi\right],
$$




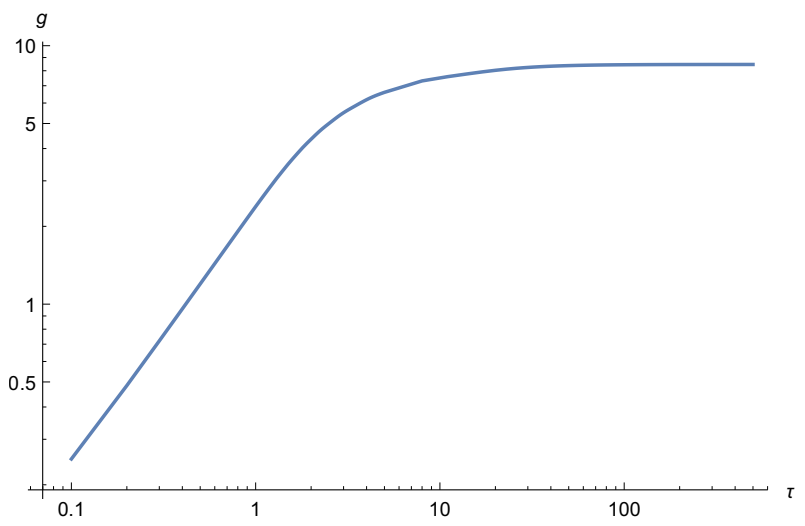

Figure 3. Plot of the spectral form factor for the Bessel case as a function of $\tau=\hbar t$ for $\hbar=$ $1 / 30, \beta=1$.

where $H$ is given by

$$
H=-\frac{\hbar^{2}}{2} \partial_{x}^{2}-u(x)
$$

In terms of the energy eigenstate $H|E\rangle=E|E\rangle(4.22)$ is written as

$$
\left\langle Z\left(\beta_{1}\right) Z\left(\beta_{2}\right)\right\rangle_{c}=\int_{0}^{1} d x \int_{1}^{\infty} d y \int_{0}^{\infty} d E_{1} \int_{0}^{\infty} d E_{2} e^{-\beta_{1} E_{1}-\beta_{2} E_{2}}\left\langle x \mid E_{1}\right\rangle\left\langle E_{1} \mid y\right\rangle\left\langle y \mid E_{2}\right\rangle\left\langle E_{2} \mid x\right\rangle .
$$

Finally, using the Laplace transform of the matrix element

$$
\int_{0}^{\infty} d E e^{-\beta E}\langle x \mid E\rangle\langle E \mid y\rangle=\frac{\sqrt{x y}}{\hbar^{2} \beta} e^{-\frac{x^{2}+y^{2}}{2 \beta \hbar^{2}}} I_{0}\left(\frac{x y}{\hbar^{2} \beta}\right)
$$

eq. (4.24) is written as

$$
\left\langle Z\left(\beta_{1}\right) Z\left(\beta_{2}\right)\right\rangle_{c}=\int_{0}^{1} d x \int_{1}^{\infty} d y \frac{x y}{\hbar^{4} \beta_{1} \beta_{2}} \exp \left[-\frac{\left(\beta_{1}+\beta_{2}\right)\left(x^{2}+y^{2}\right)}{2 \hbar^{2} \beta_{1} \beta_{2}}\right] I_{0}\left(\frac{x y}{\hbar^{2} \beta_{1}}\right) I_{0}\left(\frac{x y}{\hbar^{2} \beta_{2}}\right) .
$$

We could not find a closed form expression of this integral. By numerically evaluating the integral (4.26), in figure 3 we plot the spectral form factor defined by

$$
g(\beta, t, \hbar)=\langle Z(\beta+\mathrm{i} t) Z(\beta-\mathrm{i} t)\rangle_{c} .
$$

One can see from figure 3 that the spectral form factor exhibits the ramp and the plateau behavior as expected for a chaotic system [49-51] such as the Sachdev-Ye-Kitaev model $[52,53]$ and JT gravity.

\section{Low temperature expansion}

Let us go back to the JT supergravity case and consider the one-point function $\langle Z(\beta)\rangle$. From the result in the Bessel case (4.11) it is natural to consider the low temperature regime

$$
\hbar \ll 1, \quad \beta \gg 1 \text { with } \sigma=\frac{1}{\hbar^{2} \beta} \text { : fixed. }
$$


A slightly different low temperature regime (designated as "the 't Hooft limit") was considered in the case of bosonic JT gravity in [11, 12]. The scale of temperature $T \sim \hbar^{2}$ for $\sigma \sim \mathcal{O}(1)$ in (5.1) appears as the characteristic temperature where the contribution from the hard edge becomes of order one:

$$
\int d E \rho(E) e^{-\beta E} \sim \int d E \frac{1}{\hbar \sqrt{E}} e^{-\beta E} \sim \frac{1}{\hbar \beta^{\frac{1}{2}}} \sim \mathcal{O}(1) .
$$

At present we do not understand the physical origin of this scale $T \sim \hbar^{2}$ from the bulk JT supergravity picture. It would be interesting to understand this better.

In this low temperature regime, the genus-zero part $\langle Z(\beta)\rangle^{g=0}$ in (2.10) is expanded as

$$
\langle Z(\beta)\rangle^{g=0}=\sqrt{\frac{\sigma}{2 \pi}} e^{\hbar^{2} \sigma}=\frac{1}{\sqrt{2 \pi}} \sum_{\ell=0}^{\infty} \frac{\hbar^{2 \ell}}{\ell !} \sigma^{\ell+\frac{1}{2}} .
$$

On the other hand, the contributions from genus $g \geq 1$ become

$$
\begin{aligned}
\langle Z(\beta)\rangle^{g \geq 1} & =-\sqrt{\frac{\beta}{2 \pi}} \sum_{g=1}^{\infty} \hbar^{2 g-1} \int_{\overline{\mathcal{M}}_{g, 1}} \Theta_{g, 1} \frac{e^{\kappa}}{1-\beta \psi_{1}} \\
& =-\frac{1}{\sqrt{2 \pi}} \sum_{g=1}^{\infty} \sum_{\ell=0}^{g-1} \frac{\hbar^{2 \ell}}{\ell !} \sigma^{\ell+\frac{1}{2}-g} \int_{\overline{\mathcal{M}}_{g, 1}} \Theta_{g, 1} \kappa^{\ell} \psi_{1}^{g-1-\ell} .
\end{aligned}
$$

Here we have used the selection rule (2.5). Thus, for $\nu=0$, we find that the one-point function is expanded as

$$
\langle Z(\beta)\rangle=-\frac{1}{\sqrt{2 \pi}} \sum_{\ell=0}^{\infty} \sum_{g=0}^{\infty} \hbar^{2 \ell} \sigma^{\ell+\frac{1}{2}-g} c_{\ell}(g) .
$$

From $(3.45), c_{\ell}(g)$ is written as

$$
c_{\ell}(g)=\left.\partial_{g-1-\ell} F_{g}\right|_{t_{n}=\gamma_{n}} .
$$

We have computed $c_{\ell}(g)$ using our data of BGW free energy $F_{g}$ up to $g=30$, from which we conjecture the all genus expression of $c_{\ell}(g)$

$$
\begin{aligned}
c_{0}(g)= & \frac{(2 g-1) ! !(2 g-3) ! !}{8^{g} g !}, \\
c_{1}(g)= & \frac{((2 g-3) ! !)^{2}}{8^{g} g !} \frac{(-1+g)\left(3-4 g+8 g^{2}\right)}{3}, \\
c_{2}(g)= & \frac{((2 g-5) ! !)^{2}}{8^{g} g !} \frac{(-2+g)(-1+g)}{180} \\
& \times\left(-405+2988 g-4176 g^{2}+5632 g^{3}-4224 g^{4}+1280 g^{5}\right), \\
c_{3}(g)= & \frac{((2 g-7) ! !)^{2}}{8^{g} g !} \frac{(-3+g)(-2+g)(-1+g)}{11340}\left(70875-108270 g+4601928 g^{2}\right. \\
& \left.-6412592 g^{3}+7178816 g^{4}-6335744 g^{5}+3646976 g^{6}-1132544 g^{7}+143360 g^{8}\right) .
\end{aligned}
$$


Although we do not have a proof of (5.7), the agreement of (5.7) and the derivative of free energy (5.6) up to $g=30$ gives a strong support for this conjecture (5.7). Note that the above $c_{\ell}(g)$ correctly reproduces the expansion of the disk partition function in (5.3)

$$
-c_{\ell}(g=0)=\frac{1}{\ell !}
$$

which is parallel to the case of bosonic JT gravity [11].

Let us consider the resummation of $c_{\ell}(g)$. For $\ell=0$, the all-genus resummation of $c_{0}(g)$ is already obtained in (4.15) in terms of the modified Bessel function. We expect that the resummation of $c_{\ell}(g)$ is expressed as a certain combination of the modified Bessel functions. From the asymptotic expansion of the modified Bessel function

$$
I_{\nu}(z) \approx \frac{e^{z}}{\sqrt{2 \pi z}} \sum_{k=0}^{\infty} \frac{B_{k}}{k !(8 z)^{k}}
$$

with $B_{k}$ defined in (3.14), we find that for $\nu=0$ the one-point function in the low temperature regime (5.1) is expanded as

$$
\langle Z(\beta)\rangle=\sum_{\ell=0}^{\infty} \hbar^{2 \ell} e^{-\sigma}\left[a_{\ell}(\sigma) I_{0}(\sigma)+b_{\ell}(\sigma) I_{1}(\sigma)\right] .
$$

By matching the asymptotic expansion (5.9) with the all-genus result in (5.7), we find the first few terms of $a_{\ell}$ and $b_{\ell}$

$$
\begin{aligned}
& a_{0}=\frac{\sigma}{2}, \\
& b_{0}=\frac{\sigma}{2}, \\
& a_{1}=\frac{\sigma}{8}+\sigma^{2}-\frac{2 \sigma^{3}}{3}, \\
& b_{1}=\frac{\sigma^{2}}{3}+\frac{2 \sigma^{3}}{3} \text {, } \\
& a_{2}=\frac{15 \sigma}{256}-\frac{5 \sigma^{2}}{64}+\frac{137 \sigma^{3}}{120} \\
& b_{2}=\frac{43 \sigma^{2}}{960}+\frac{\sigma^{3}}{40} \\
& -\frac{21 \sigma^{4}}{5}+\frac{28 \sigma^{5}}{5}-\frac{16 \sigma^{6}}{9}, \\
& +\frac{31 \sigma^{4}}{15}-\frac{212 \sigma^{5}}{45}+\frac{16 \sigma^{6}}{9} .
\end{aligned}
$$

We emphasize that $a_{\ell}(\sigma)$ and $b_{\ell}(\sigma)$ contain the all-genus information of the intersection numbers $\int_{\overline{\mathcal{M}}_{g, 1}} \Theta_{g, 1} \kappa^{\ell} \psi^{g-1-\ell}$ at the fixed number of $\kappa$-insertions. ${ }^{4}$

It is straightforward to generalize this computation to the non-zero $\nu$ case. For $\nu \neq 0$ we find that the one-point function is expanded as

$$
\langle Z(\beta)\rangle=\langle Z(\beta)\rangle_{\text {Bessel }}+\sum_{\ell=1}^{\infty} \hbar^{2 \ell} e^{-\sigma}\left[a_{\ell}(\sigma) I_{\nu}(\sigma)+b_{\ell}(\sigma) I_{\nu-1}(\sigma)\right]
$$

\footnotetext{
${ }^{4}$ We have computed $a_{\ell}(\sigma), b_{\ell}(\sigma)$ up to $\ell=7$. These data are available upon request to the authors.
} 
where the $\ell=0$ term is given by the Bessel case (4.10). By matching the asymptotic expansion of $I_{\nu}(\sigma), I_{\nu-1}(\sigma)$ and the genus expansion of $\langle Z(\beta)\rangle$ at $\nu \neq 0$, we find

$$
\begin{aligned}
a_{1}(\sigma)= & \frac{4 \nu^{2}-8 \nu+3}{24} \sigma+\frac{1}{3}(3-2 \nu) \sigma^{2}-\frac{2 \sigma^{3}}{3} \\
b_{1}(\sigma)= & \frac{1}{3} \sigma^{2}+\frac{2 \sigma^{3}}{3}, \\
a_{2}(\sigma)= & \frac{-320 \nu^{5}+304 \nu^{4}+2144 \nu^{3}-2776 \nu^{2}-516 \nu+675}{11520} \sigma \\
& +\frac{560 \nu^{4}-2016 \nu^{3}+1912 \nu^{2}-72 \nu-225}{2880} \sigma^{2}+\frac{1}{120}\left(92 \nu^{2}-248 \nu+137\right) \sigma^{3} \\
& +\frac{1}{45}\left(-40 \nu^{2}+212 \nu-189\right) \sigma^{4}-\frac{4}{45}(20 \nu-63) \sigma^{5}-\frac{16 \sigma^{6}}{9}, \\
b_{2}(\sigma)= & \frac{80 \nu^{4}-536 \nu^{2}+129}{2880} \sigma^{2}+\frac{1}{40}\left(28 \nu^{2}+1\right) \sigma^{3}+\frac{31 \sigma^{4}}{15}-\frac{212 \sigma^{5}}{45}+\frac{16 \sigma^{6}}{9} .
\end{aligned}
$$

If we set $\nu=0$ this reduces to (5.11) as expected.

In the case of bosonic JT gravity, we observed that the free energy $\log \langle Z(\beta)\rangle$ in the low temperature regime has an intimate connection to the integral $\int x d y$ on the spectral curve $y=\frac{1}{2} \sin (2 z)[11,12]$. On the other hand, in the present case of JT supergravity we could not find a simple relation between the free energy $\log \langle Z(\beta)\rangle$ and the spectral curve $y=-\frac{\cos (2 z)}{\sqrt{2} z}$. In the next section, we will see that in the large $\nu$ regime (1.1) the relation to the spectral curve becomes more transparent.

\section{Large $\nu$ regime}

In this section we consider the large $\nu$ regime (1.1). This regime was considered before in the BGW model in [19] and in the minimal superstring in [35]. One interesting property of this regime is that the free energy of BGW model admits the genus expansion

$$
F=\sum_{g=0}^{\infty} \hbar^{2 g-2} \mathcal{F}_{g}\left(q,\left\{t_{k}\right\}\right)
$$

with non-zero genus-zero term $\mathcal{F}_{0} \neq 0$. This is in contrast to the case of finite $\nu$ where the genus-zero free energy vanishes $F_{0}=0$. We stress that the two statements $F_{0}=0$ and $\mathcal{F}_{0} \neq 0$ are not in contradiction. Let us see this by an example. In the large $\nu$ limit (1.1), the genus-one free energy $F_{1}$ in (3.15) becomes

$$
F_{1}=-\frac{1-4 \nu^{2}}{8} \log \left(1-t_{0}\right)=\frac{q^{2}}{2 \hbar^{2}} \log \left(1-t_{0}\right)-\frac{1}{8} \log \left(1-t_{0}\right) .
$$

The first term on the right hand side of (6.2) is order $\mathcal{O}\left(\hbar^{-2}\right)$ and it can be thought of as a part of the genus-zero free energy $\mathcal{F}_{0}$. Although the genus-zero term vanishes $F_{0}=0$ in the original finite $\nu$ case, after taking the scaling limit (1.1), the order $\nu^{2 g}$ term in the genus- $g$ free energy $F_{g}(g \geq 1)$ contributes to the genus-zero free energy $\mathcal{F}_{0}$ as we have seen in $(6.2)$. 
From the result of free energy at non-zero $\nu$ in (3.15) and (3.16), we find that

$$
\mathcal{F}_{0}=\frac{q^{2}}{2} \log \left(1-t_{0}\right)+\sum_{\substack{j_{a} \geq 0 \\ \sum_{a} j_{a}=m \geq 1 \\ \sum_{a} a j_{a}=n}} \frac{(2 n+m-1) !}{(2 n+2) !} \frac{\left(-q^{2}\right)^{n+1}}{2^{n}\left(1-t_{0}\right)^{2 n+m}} \prod_{a=1}^{\infty} \frac{t_{a}^{j_{a}}}{a ! j_{a} j_{a} !} .
$$

Note that the first term of $\mathcal{F}_{0}$ comes from $F_{1}$ in (6.2) and the rest of (6.3) comes from $F_{g}(g \geq 2)$. This expression (6.3) was originally obtained in [19]. We will see that the higher genus corrections $\mathcal{F}_{g}(g \geq 1)$ are determined from the genus-zero data only, which is known as the constitutive relation [20].

Before moving on, let us comment on the physical meaning of the scaling limit (1.1). As discussed in [35], in the limit (1.1) the factor $\lambda^{\alpha}=\lambda^{1+2 \nu}$ in the measure of the eigenvalue integral contributes to the effective potential as $e^{-\frac{1}{\hbar} V_{\text {eff }}(\lambda)}=e^{\frac{2 q}{\hbar} \log \lambda}$, i.e. the model acquires the logarithmic potential like the Penner model. This modification of the potential changes the behavior of free energy qualitatively, e.g. $F_{0}=0$ to $\mathcal{F}_{0} \neq 0$. We do not understand the bulk gravitational interpretation of this effect. It would be interesting to clarify the bulk interpretation of this limit (1.1).

\subsection{Genus-zero part}

Let us take a closer look at the genus-zero part (6.3). From (6.3) the genus-zero potential $u_{0}=\partial_{0}^{2} \mathcal{F}_{0}$ is given by

$$
u_{0}=\sum_{\substack{j_{a} \geq 0 \\ \sum_{a} j_{a}=m \\ \sum_{a} a j_{a}=n}} \frac{(2 n+m+1) !}{(2 n+2) !} \frac{\left(-q^{2}\right)^{n+1}}{2^{n}\left(1-t_{0}\right)^{2 n+m+2}} \prod_{a=1}^{\infty} \frac{t_{a}^{j_{a}}}{a ! j_{a}} j_{a} !
$$

One can check that this satisfies the genus-zero version of the string equation (3.17)

$$
u_{0}=-\frac{q^{2}}{2\left(1-I_{0}\right)^{2}}
$$

where we introduced $I_{n}\left(u_{0}\right)$ by

$$
I_{n}\left(u_{0}\right)=\sum_{k=0}^{\infty} t_{n+k} \frac{u_{0}^{k}}{k !} .
$$

These variables $\left\{I_{n}\right\}$ are introduced by Itzykson and Zuber [54] in the context of KW model. Note that in the KW model the genus-zero string equation reads

$$
u_{0}=I_{0}\left(u_{0}\right)
$$

As in the case of KW model, the genus-zero free energy $\mathcal{F}_{0}$ in the BGW model can be written as an integral of $I_{0}$. To see this let us rewrite (6.5) as

$$
I_{0}\left(u_{0}\right)-1+\frac{q}{\sqrt{-2 u_{0}}}=0
$$


Here we assumed $q>0, u_{0}<0$ and took the appropriate branch of the square root. Then we find that $\mathcal{F}_{0}$ is written as

$$
\begin{aligned}
\mathcal{F}_{0}= & \lim _{\epsilon \rightarrow+0} \frac{1}{2} \int_{-\epsilon}^{u_{0}} d u\left(I_{0}(u)-1+\frac{q}{\sqrt{-2 u}}\right)^{2} \\
& +\frac{q^{2}}{2} \log q-\frac{3}{4} q^{2}-\frac{q^{2}}{4} \log \epsilon .
\end{aligned}
$$

Using the "equation of motion" (6.8) one can show that this expression satisfies the required relation $\partial_{0}^{2} \mathcal{F}_{0}=u_{0}$

$$
\partial_{0}^{2} \mathcal{F}_{0}=\lim _{\epsilon \rightarrow+0} \int_{-\epsilon}^{u_{0}} d u=u_{0}
$$

We have also checked that (6.9) reproduces (6.3) in the small $q$ expansion. The $q$-dependent constant terms in the second line of (6.9) are necessary for this agreement, and we will argue in section 6.3 that they come from the volume of unitary group $\mathrm{U}(\nu)$.

Next, let us consider the eigenvalue density at genus-zero from the perspective of the large $\nu$ regime (1.1). ${ }^{5}$ At the on-shell value of the coupling $t_{n}=\gamma_{n}(n \geq 1)$ with $t_{0} \neq 0$, the string equation (6.5) is solved as

$$
t_{0}=J_{0}\left(2 \sqrt{u_{0}}\right)-\frac{q}{\sqrt{-2 u_{0}}}
$$

where we used the relation

$$
\sum_{k=1}^{\infty} \gamma_{k} \frac{u_{0}^{k}}{k !}=\sum_{k=1}^{\infty} \frac{(-1)^{k-1} u_{0}^{k}}{k !^{2}}=1-J_{0}\left(2 \sqrt{u_{0}}\right) .
$$

From this relation (6.11), $u_{0}$ is obtained in the small $q$ expansion as

$$
u_{0}=-\frac{q^{2}}{2\left(1-t_{0}\right)^{2}}+\frac{q^{4}}{2\left(1-t_{0}\right)^{5}}-\frac{\left(13+t_{0}\right) q^{6}}{16\left(1-t_{0}\right)^{8}}+\mathcal{O}\left(q^{8}\right) .
$$

Now let us recall that the one-point function is written as the expectation value of the macroscopic loop operator [11]

$$
\langle Z(\beta)\rangle=\operatorname{Tr}\left(e^{-\beta H} \Pi\right)=\int_{0}^{1} d x\left\langle x\left|e^{-\beta H}\right| x\right\rangle .
$$

At the genus-zero this reduces to

$$
\langle Z(\beta)\rangle^{g=0}=\frac{1}{\sqrt{2 \pi \beta} \hbar} \int_{0}^{1} d t_{0} e^{\beta u_{0}} .
$$

As discussed in [11] it is convenient to change the integration variable from $t_{0}$ to $v=-u_{0}$. Then using the relation

$$
\int_{v}^{\infty} \frac{d E}{\sqrt{E-v}} e^{-\beta E}=\sqrt{\frac{\pi}{\beta}} e^{-\beta v},
$$

\footnotetext{
${ }^{5} \mathrm{~A}$ similar derivation of the genus-zero eigenvalue density in the case of finite $\nu$ (i.e. without the condition (1.1)) was considered earlier in [37]. To derive the eigenvalue density correctly, however, we believe it is essential to consider the large $\nu$ regime and take the limit of $q \rightarrow 0$, as we shall see later.
} 
eq. (6.15) is rewritten as the integral of eigenvalue density $\rho(E)$

$$
\langle Z(\beta)\rangle^{g=0}=\int_{E_{0}}^{\infty} d E \rho(E) e^{-\beta E},
$$

where $\rho(E)$ is given by

$$
\rho(E)=\frac{1}{\sqrt{2} \pi \hbar} \int_{E_{0}}^{E} d v \frac{1}{\sqrt{E-v}} \frac{d t_{0}}{d v} .
$$

For $v=-u_{0}, t_{0}$ in $(6.11)$ becomes

$$
t_{0}=I_{0}(2 \sqrt{v})-\frac{q}{\sqrt{2 v}},
$$

where $I_{0}(2 \sqrt{v})$ denotes the modified Bessel function of the first kind, and $E_{0}$ in (6.18) is defined by the zero of $t_{0},{ }^{6}$

$$
I_{0}\left(2 \sqrt{E_{0}}\right)-\frac{q}{\sqrt{2 E_{0}}}=0 .
$$

Then $\rho(E)$ in $(6.18)$ is written more explicitly as

$$
\rho(E)=\frac{1}{\sqrt{2} \pi \hbar} \int_{E_{0}}^{E} d v \frac{1}{\sqrt{E-v}}\left(\frac{I_{1}(2 \sqrt{v})}{\sqrt{v}}+\frac{q}{(2 v)^{\frac{3}{2}}}\right) .
$$

From (6.13), one can easily find the small $q$ expansion of $E_{0}$ as

$$
E_{0}=-\left.u_{0}\right|_{t_{0}=0}=\frac{q^{2}}{2}-\frac{q^{4}}{2}+\frac{13 q^{6}}{16}+\mathcal{O}\left(q^{8}\right) .
$$

Let us consider the $q \rightarrow 0$ limit of $\rho(E)$ in (6.21). The first term of (6.21) has a naive limit

$$
\frac{1}{\sqrt{2} \pi \hbar} \int_{0}^{E} d v \frac{1}{\sqrt{E-v}} \frac{I_{1}(2 \sqrt{v})}{\sqrt{v}}=\frac{1}{\sqrt{2} \pi \hbar} \frac{2 \sinh ^{2}(\sqrt{E})}{\sqrt{E}},
$$

while the second term of (6.21) becomes

$$
\lim _{q \rightarrow 0} \frac{1}{\sqrt{2} \pi \hbar} \int_{E_{0}}^{E} d v \frac{1}{\sqrt{E-v}} \frac{q}{(2 v)^{\frac{3}{2}}}=\lim _{q \rightarrow 0} \frac{1}{\sqrt{2} \pi \hbar} \frac{1}{\sqrt{E}} \int_{\frac{q^{2}}{2}} d v \frac{q}{(2 v)^{\frac{3}{2}}}=\frac{1}{\sqrt{2} \pi \hbar} \frac{1}{\sqrt{E}} .
$$

Putting the two contributions (6.23) and (6.24) together, we find

$$
\lim _{q \rightarrow 0} \rho(E)=\frac{\cosh 2 \sqrt{E}}{\sqrt{2 E} \pi \hbar}
$$

which agrees with the genus-zero eigenvalue density $\rho_{0}(E)$ in $(2.23)$ for $\nu=0$. Thus we showed that the $E^{-\frac{1}{2}}$ hard edge behavior of the eigenvalue density is correctly reproduced from $\rho(E)$ in (6.21) by carefully taking the limit $q \rightarrow 0$. A similar derivation of $\rho_{0}(E)$ was considered in [37], but the details are different from ours. In particular, neither the large

\footnotetext{
${ }^{6} \mathrm{~A}$ similar shift of threshold energy $E_{0}$ is considered in JT gravity with conical defects $[55,56]$.
} 


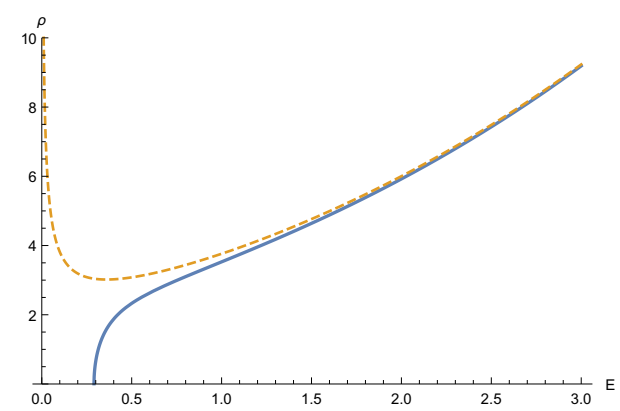

(a) $q=1$

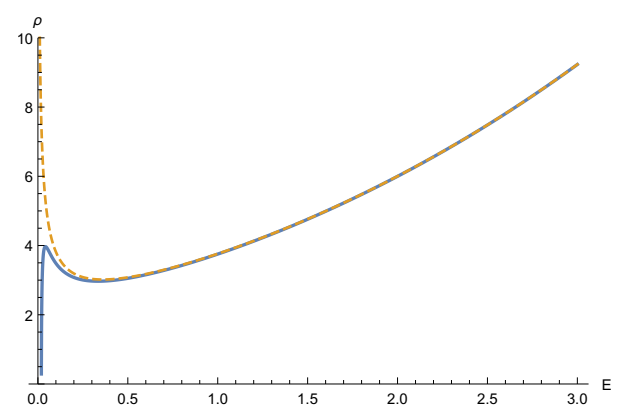

(b) $q=1 / 5$

Figure 4. Plot of the eigenvalue density for (a) $q=1$ and (b) $q=1 / 5$. The solid curve is the plot of $\sqrt{2} \pi \hbar \rho(E)$ while the dashed curve represents $\frac{\cosh (2 \sqrt{E})}{\sqrt{E}}$.

$\nu$ regime nor the limit $q \rightarrow 0$ were considered in [37] and we believe that the appearance of the $E^{-\frac{1}{2}}$ term in (6.24) was not properly explained in [37].

It is interesting to study the behavior of $\rho(E)$ at non-zero $q \cdot{ }^{7}$ In figure 4 we show the plot of (6.21) for $q=1$ and $q=1 / 5$. At finite $q, \rho(E)$ vanishes at $E=E_{0}$ as

$$
\lim _{E \rightarrow E_{0}} \rho(E) \sim \sqrt{E-E_{0}} .
$$

However, as $q \rightarrow 0 \rho(E)$ develops a hard edge $E^{-\frac{1}{2}}$ near $E=0$ as we can see from figure 4(b).

\subsection{Higher genus free energy and constitutive relation}

The large $\nu$ BGW free energy $\mathcal{F}_{g}$ as well as the $\mathrm{KW}$ free energy $F_{g}^{\mathrm{KW}}$ is a formal power series in infinitely many variables $\left\{t_{k}\right\}$. Itzykson and Zuber found that $F_{g}^{\mathrm{KW}}$ is concisely expressed when written in the variables $\left\{I_{k}\right\}$ [54]. It has been known that the free energy can also be expressed concisely in another set of variables $\left\{\partial_{0}^{k} u_{0}\right\}$ with $u_{0}=\partial_{0}^{2} F_{0}^{\mathrm{KW}}[21,57,58]$. In other words, higher genus free energies $F_{g}^{\mathrm{KW}}(g \geq 1)$ are expressed purely in terms of the genus zero data. This is known as the constitutive relation [20]. In what follows we will study the BGW free energy $\mathcal{F}_{g}$ in a similar fashion and elucidate the constitutive relation for the BGW model.

As mentioned above, $F_{g}^{\mathrm{KW}}$ are expressed in any of the bases, $\left\{t_{k}\right\},\left\{I_{k}\right\}$ and $\left\{\partial_{0}^{k} u_{0}\right\}$. The explicit transformation formulas among these variables are known [57]. The advantage of the expressions in the latter two bases is that one can determine $F_{g}^{\mathrm{KW}}$ iteratively by merely solving the $\mathrm{KdV}$ equation

$$
\partial_{1} u=\partial_{0}\left[\frac{u^{2}}{2}+\frac{\hbar^{2} \partial_{0}^{2} u}{12}\right]
$$

with $u=\hbar^{2} \partial_{0}^{2} F^{\mathrm{KW}}$. Since $\hbar^{2} \partial_{0}^{2} F^{\mathrm{BGW}}$ also satisfies the same $\mathrm{KdV}$ equation, it is natural to expect that $\mathcal{F}_{g}$ can also be determined in this way if it is expressed in a suitable basis.

\footnotetext{
${ }^{7}$ In a recent paper [38] the eigenvalue density is studied numerically at $\nu= \pm \frac{1}{2}$.
} 
Let us therefore assume that $\mathcal{F}_{g}$ is expressed in the basis $\left\{\partial_{0}^{k} u_{0}\right\}$ with $u_{0}$ given in (6.4) and try to determine it by solving (6.27). This does not necessarily mean that we have to work out the full change of variables from $\left\{t_{k}\right\}$ to $\left\{\partial_{0}^{k} u_{0}\right\}$. Since only derivatives with respect to $t_{0}, t_{1}$ appear in (6.27), for the purpose of calculating $\mathcal{F}_{g}$ we have only to treat $t_{0}$ and $t_{1}$ as variables and can regard $\left\{t_{k}\right\}(k \geq 2)$ as parameters. From this point of view it is sufficient to consider the change of variables from $\left(t_{0}, t_{1}\right)$ to

$$
u_{0}=: y, \quad \partial_{0} u_{0}=: t^{-1}
$$

with $\left\{t_{k}\right\}(k \geq 2)$ held fixed.

For our later purposes, it is convenient to express the genus zero string equation in the abstract form

$$
I_{0}\left(u_{0}\right)=\varphi\left(u_{0}\right)
$$

For the KW and BGW models the function $\varphi\left(u_{0}\right)$ is given respectively by

$$
\varphi_{\mathrm{KW}}\left(u_{0}\right)=u_{0}, \quad \varphi_{\mathrm{BGW}}\left(u_{0}\right)=1-\frac{q}{\sqrt{-2 u_{0}}} .
$$

By differentiating (6.29) in $t_{0}$ we have

$$
1+\left(\partial_{0} u_{0}\right) I_{1}\left(u_{0}\right)=\left(\partial_{0} u_{0}\right) \varphi^{\prime}\left(u_{0}\right)
$$

It then follows that

$$
t=\varphi^{\prime}\left(u_{0}\right)-I_{1}\left(u_{0}\right)
$$

We next introduce

$$
\tilde{B}_{-1}(v):=-\varphi(v)+\sum_{k=2}^{\infty} t_{k} \frac{v^{k}}{k !}
$$

and define $\tilde{B}_{n}(v)(n \geq 0)$ by the relation

$$
\tilde{B}_{n}=-\partial_{v} \tilde{B}_{n-1}, \quad n \geq 0 .
$$

$\tilde{B}_{n}$ are related to $I_{n}$ in (6.6) as

$$
\begin{aligned}
\tilde{B}_{-1}(v) & =-\varphi(v)-t_{0}-t_{1} v+I_{0}(v), \\
\tilde{B}_{0}(v) & =\varphi^{\prime}(v)+t_{1}-I_{1}(v), \\
\tilde{B}_{n}(v) & =(-1)^{n+1} \tilde{I}_{n+1}(v) \quad(n \geq 1)
\end{aligned}
$$

with

$$
\tilde{I}_{n}(v):=I_{n}(v)-\varphi^{(n)}(v) \quad(n \geq 2) .
$$

It is clear either from (6.6) or from (6.34)-(6.35) that

$$
\partial_{v} \tilde{I}_{k}(v)=\tilde{I}_{k+1}(v) .
$$


Note that $\tilde{B}_{n}$ is a natural generalization of $B_{n}$ used in our previous work [11, 12]: $\tilde{B}_{n}$ satisfies the same relation (6.34) as $B_{n}$ and $\tilde{B}_{n}$ with $\varphi=\varphi_{\mathrm{KW}}$ reduces to $B_{n}$ by setting $t_{k}=\frac{(-1)^{k}}{(k-1) !}(k \geq 2)$. In the same sense $\tilde{I}_{n}(n \geq 2)$ is a natural generalization of $I_{n}$ for the KW model: $\tilde{I}_{n}$ satisfies (6.37) and $\tilde{I}_{n}$ with $\varphi=\varphi_{\mathrm{KW}}$ coincides with $I_{n}$.

The relations (6.29) and (6.32) are written in terms of $\tilde{B}_{n}$ as

$$
t_{1}=\tilde{B}_{0}(y)-t, \quad t_{0}=-\tilde{B}_{-1}(y)-y t_{1} .
$$

From these relations one can derive

$$
\partial_{0}=\frac{1}{t}\left(\partial_{y}-\tilde{B}_{1}(y) \partial_{t}\right), \quad \partial_{1}=y \partial_{0}-\partial_{t} .
$$

This is formally identical to the change of variables originally introduced by Zograf [13] and used in our previous work $[11,12]$. However, we stress that it is now valid for both KW and BGW models with full dependence on $t_{k}(k \geq 2)$ being incorporated. By rewriting $\partial_{0}$ in (6.39) as

$$
\partial_{0}=\frac{1}{t}\left(\partial_{y}-\tilde{I}_{2}(y) \partial_{t}\right)
$$

and applying it repeatedly on (6.28) one obtains

$$
\partial_{0}^{2} u_{0}=\frac{\tilde{I}_{2}}{t^{3}}, \quad \partial_{0}^{3} u_{0}=\frac{\tilde{I}_{3}}{t^{4}}+\frac{3 \tilde{I}_{2}^{2}}{t^{5}}, \quad \partial_{0}^{4} u_{0}=\frac{\tilde{I}_{4}}{t^{5}}+\frac{10 \tilde{I}_{2} \tilde{I}_{3}}{t^{6}}+\frac{15 \tilde{I}_{2}^{3}}{t^{7}}, \quad \cdots
$$

In general, one can prove that

$$
\partial_{0}^{n} u_{0}=\sum_{\substack{m_{j} \geq 0 \\ \sum_{j \geq 1} j m_{j}=n-1}} \frac{\left(\sum_{j}(j+1) m_{j}\right) !}{\prod_{j}((j+1) !)^{m_{j}} m_{j} !} \cdot \frac{\prod_{j} \tilde{I}_{j+1}^{m_{j}}}{t^{\sum_{j}(j+1) m_{j}+1}} .
$$

This is a straightforward generalization of the formula for the KW model originally obtained in [57]. Again, this formula is now valid for both KW and BGW models at arbitrary values of $t_{k}$. Note that (6.42) is inverted as [57]

$$
\tilde{I}_{n}=-\sum_{\substack{m_{j} \geq 0 \\ \sum_{j \geq 1} j m_{j}=n-1}} \frac{\left(\sum_{j}(j+1) m_{j}\right) !}{\prod_{j}((j+1) !)^{m_{j}} m_{j} !} \cdot \frac{\prod_{j}\left(-\partial_{0}^{j+1} u_{0}\right)^{m_{j}}}{\left(\partial_{0} u_{0}\right)^{\sum_{j}(j+1) m_{j}+1}} .
$$

With these preparations we can solve the $\mathrm{KdV}$ equation and calculate $\mathcal{F}_{g}$ iteratively in the same way as in the KW case. As a demonstration let us calculate the genus one free energy along the lines of [54]. By plugging the expansion

$$
u=\sum_{g=0}^{\infty} \hbar^{2 g} u_{g}
$$

into the $\mathrm{KdV}$ equation (6.27), one obtains

$$
\partial_{0}\left[\left(\partial_{1}-u_{0} \partial_{0}\right) \partial_{0} \mathcal{F}_{1}-\frac{1}{12} \partial_{0}^{2} u_{0}\right]=0
$$


at the order of $\hbar^{2}$. From (6.41) and (6.40) one sees that

$$
\partial_{0}^{2} u_{0}=\frac{\tilde{I}_{2}(y)}{t^{3}}=\partial_{t}\left(-\frac{\tilde{I}_{2}(y)}{2 t^{2}}\right)=\frac{1}{2} \partial_{t} \partial_{0} \log t .
$$

Using this and the second relation in (6.39) one can rewrite (6.45) as

$$
\partial_{0} \partial_{t} \partial_{0}\left(\mathcal{F}_{1}+\frac{1}{24} \log t\right)=0 \text {. }
$$

This suggests that

$$
\mathcal{F}_{1}=-\frac{1}{24} \log t+\text { const. }
$$

Indeed, by means of small $q$ expansion we explicitly verified that

$$
\begin{aligned}
\mathcal{F}_{1} & =-\frac{1}{24} \log t-\frac{1}{12} \log q \\
& =\frac{1}{24} \log \left(\partial_{0} u_{0}\right)-\frac{1}{12} \log q .
\end{aligned}
$$

Similarly, one can compute the higher genus free energy $\mathcal{F}_{g}(g \geq 2)$ up to an integration constant which cannot be constrained by the KdV equation. By means of small $q$ expansion we determined the constant term and verified that

$$
\begin{aligned}
\mathcal{F}_{2} & =\frac{\tilde{I}_{4}}{1152 t^{3}}+\frac{29 \tilde{I}_{2} \tilde{I}_{3}}{5760 t^{4}}+\frac{7 \tilde{I}_{2}^{3}}{1440 t^{5}}-\frac{1}{240 q^{2}} \\
& =\frac{\partial_{0}^{4} u_{0}}{1152\left(\partial_{0} u_{0}\right)^{2}}-\frac{7 \partial_{0}^{2} u_{0} \partial_{0}^{3} u_{0}}{1920\left(\partial_{0} u_{0}\right)^{3}}+\frac{\left(\partial_{0}^{2} u_{0}\right)^{3}}{360\left(\partial_{0} u_{0}\right)^{4}}-\frac{1}{240 q^{2}} .
\end{aligned}
$$

The results (6.49)-(6.50) should be compared with the well-known results of the KW free energies [54]

$$
\begin{aligned}
F_{1}^{\mathrm{KW}} & =-\frac{1}{24} \log t \\
F_{2}^{\mathrm{KW}} & =\frac{I_{4}}{1152 t^{3}}+\frac{29 I_{2} I_{3}}{5760 t^{4}}+\frac{7 I_{2}^{3}}{1440 t^{5}} .
\end{aligned}
$$

One immediately finds that the expressions of $\mathcal{F}_{g}$ and $F_{g}^{\mathrm{KW}}$ are essentially identical up to the constant term. This is expected because both free energies are determined by the same KdV equation with the common expression of differential operators (6.39) and all $\tilde{I}_{k}$ 's appearing in these expressions are originated from $\tilde{B}_{1}=\tilde{I}_{2}$ in the expression of $\partial_{0}$. We conjecture that this correspondence holds for any $g$.

Assuming that this conjecture holds, let us discuss the constitutive relation for the BGW model. Since the transformation formula $(6.42)$ is common to both models, $\mathcal{F}_{g}$ and $F_{g}^{\mathrm{KW}}$ take, up to the constant term, exactly the same form when expressed in the $\left\{\partial_{0}^{k} u_{0}\right\}$ basis. This means that the constitutive relations for the KW and BGW models are identical. Our discussion is based only on the KdV equation (6.27), the string equation of the form (6.29) and the assumption that the free energy can be expressed in the basis 
$\left\{\partial_{0}^{k} u_{0}\right\}$. Thus, we may conclude that the constitutive relation for the $\mathrm{KW}$ model is not specific to the model, but rather a universal property of the tau-function for the $\mathrm{KdV}$ hierarchy.

Let us present our conjecture more explicitly. We conjecture that $\mathcal{F}_{g}(g \geq 2)$ is written in the form

$$
\begin{aligned}
\mathcal{F}_{g}= & \sum_{\substack{l_{j} \geq 0 \\
\sum_{j=2}^{3 g-2}(j-1) l_{j}=3 g-3}} b_{l_{2} \cdots l_{3 g-2}} \frac{\tilde{I}_{2}^{l_{2}} \cdots \tilde{I}_{3 g-2}^{l_{3 g-2}}}{t^{2(g-1)+\sum_{j=2}^{3 g-2} l_{j}}}+c_{g} q^{2-2 g} \\
= & \sum_{\substack{l_{j} \geq 0 \\
\sum_{j=2}^{3 g-2}(j-1) l_{j}=3 g-3}} a_{l_{2} \cdots l_{3 g-2}} \frac{\left(\partial_{0}^{2} u_{0}\right)^{l_{2}} \cdots\left(\partial_{0}^{3 g-2} u_{0}\right)^{l_{3 g-2}}}{\left(\partial_{0} u_{0}\right)^{2(1-g)+\sum_{j=2}^{3 g-2} j l_{j}}}+c_{g} q^{2-2 g} .
\end{aligned}
$$

Note that with $\varphi=\varphi_{\mathrm{KW}}$ and without the constant term $c_{g} q^{2-2 g}$, this is exactly the theorem proved in $[21,58]$ for the KW model. (The first expression for the KW model was originally conjectured in [54].) Once $\mathcal{F}_{g}$ is assumed in this form, one can fully determine the coefficients $a_{l_{2} \cdots l_{3 g-2}}$ and $b_{l_{2} \cdots l_{3 g-2}}$ by the KdV equation (6.27). Their values are in fact common to both KW and BGW models. We will also conjecture about the constant term $c_{g} q^{2-2 g}$ in the next subsection.

Let us make a few comments towards a proof of the conjecture. In the case of the KW model, the key ingredients of the proof in [21] are the degree counting and the Virasoro condition $\mathcal{L}_{-1} e^{F^{\mathrm{KW}}}=0$. In the BGW case the degree counting can be done in the same way as follows: we see from (3.11)-(3.12) that we can assign degree $1-k$ to $t_{k}-\delta_{k, 0}$ so that $\mathcal{F}_{g}$ is of degree $3 g-3$. This degree assignment works consistently in the presence of $\nu$ as well ( $\nu$ should be of degree 0 ). By further assigning degree $\frac{3}{2}$ to $\hbar$, we have

$$
[q]=\frac{3}{2}, \quad\left[F^{\mathrm{BGW}}\right]=0, \quad\left[\mathcal{F}_{g}\right]=3 g-3, \quad\left[\partial_{0}^{k} u_{0}\right]=1-k, \quad\left[I_{k}\left(u_{0}\right)-\delta_{k, 0}\right]=1-k,
$$

where [.] denotes the degree. This is the same degree assignment as in the case of $F^{\mathrm{KW}}[21]$. On the other hand, the Virasoro $\mathcal{L}_{-1}$ condition ensures that $F_{g}^{\mathrm{KW}}(g \geq 1)$ does not depend on $u_{0}$ when written in the $\left\{\partial_{0}^{k} u_{0}\right\}$ basis. However, such a condition seems to be absent in the BGW model (see (3.33)). It is therefore not clear to us how to prove the $u_{0}$-independence of $\mathcal{F}_{g}(g \geq 1)$. If one can prove this $u_{0}$-independence in some way, the rest of the structure in (6.52) can be shown in the same manner as in [21] based on the degree counting.

To summarize, many of the results about the KW model that are obtained by solving the $\mathrm{KdV}$ equation are immediately promoted to the universal results by the mere replacement

$$
I_{0} \rightarrow u_{0}, \quad 1-I_{1} \rightarrow t=\varphi^{\prime}-I_{1}, \quad I_{k} \rightarrow \tilde{I}_{k}(k \geq 2), \quad B_{k} \rightarrow \tilde{B}_{k}(k \geq 1)
$$

We stress that the universal results are valid for both KW and BGW models at arbitrary values of $t_{k}$. For instance, we can apply this to the results about the $n$-point correlators $\left\langle Z\left(\beta_{1}\right) \cdots Z\left(\beta_{n}\right)\right\rangle_{c}$ and the Baker-Akhiezer function obtained in our previous work [11, 12]. 


\subsection{Volume of $\mathrm{U}(\nu)$}

We conjecture that the extra $q$-dependent constant comes from the volume of the unitary group $\mathrm{U}(\nu)[59]$

$$
-\log [\operatorname{vol} \mathrm{U}(\nu)]=\log \frac{G_{2}(\nu+1)}{(2 \pi)^{\frac{1}{2} \nu(\nu+1)}},
$$

where $G_{2}(z)$ denotes the Barnes $G$-function. In the large $\nu$ regime this is expanded as

$$
\begin{aligned}
-\log [\operatorname{vol} \mathrm{U}(\nu)] & =\frac{\nu^{2}}{2} \log \frac{\nu}{2 \pi}-\frac{3}{4} \nu^{2}-\frac{1}{12} \log \nu+\zeta^{\prime}(-1)+\sum_{g \geq 2} \frac{B_{2 g}}{2 g(2 g-2)} \nu^{2-2 g} \\
& =: \sum_{g=0}^{\infty} \hbar^{2 g-2} \mathcal{F}_{g}^{\mathrm{vol}},
\end{aligned}
$$

where the genus- $g$ free energy $\mathcal{F}_{g}^{\mathrm{vol}}$ coming from the volume of $\mathrm{U}(\nu)$ is given by

$$
\begin{aligned}
\mathcal{F}_{0}^{\mathrm{vol}} & =\frac{q^{2}}{2} \log \frac{q}{2 \pi \hbar}-\frac{3}{4} q^{2}, \\
\mathcal{F}_{1}^{\mathrm{vol}} & =-\frac{1}{12} \log \frac{q}{\hbar}+\zeta^{\prime}(-1), \\
\mathcal{F}_{g}^{\mathrm{vol}} & =\frac{B_{2 g}}{2 g(2 g-2)} q^{2-2 g} \quad(g \geq 2) .
\end{aligned}
$$

Here $B_{2 g}$ denotes the Bernoulli number. One can see that the genus zero part $\mathcal{F}_{0}^{\mathrm{vol}}$ agrees with the second line of (6.9) under the identification $\sqrt{\epsilon}=2 \pi \hbar$. We observe that $\mathcal{F}_{g}^{\mathrm{vol}}(g \geq$ 2) has a negative power of $q$. On the other hand, the free energy $\mathcal{F}_{g}$ of BGW model in the large $\nu$ regime (1.1) is a smooth function of $q$ and it contains only the positive power of $q$. What is happening is that the negative power of $q$ coming from the constitutive part $\mathcal{F}_{g}^{\text {con }}$ is exactly canceled by $\mathcal{F}_{g}^{\text {vol }}$. It turns out that such a term arises by substituting the leading term in the small $q$ expansion of $u_{0}$ in (6.13)

$$
\left.\mathcal{F}_{g}^{\mathrm{con}}\right|_{u_{0}=-\frac{q^{2}}{2\left(1-t_{0}\right)^{2}}}=-\mathcal{F}_{g}^{\mathrm{vol}}
$$

We have checked this relation up to $g=20 .{ }^{8}$ Then the free energy of BGW model (6.52) is free from the negative power of $q$

$$
\mathcal{F}_{g}=\mathcal{F}_{g}^{\mathrm{con}}+\mathcal{F}_{g}^{\mathrm{vol}}
$$

\subsection{Correlators at large $\nu$}

From the result of free energy $F_{g}$ at non-zero $\nu$, one can compute the connected correlator $\left\langle Z\left(\beta_{1}\right) \cdots Z\left(\beta_{n}\right)\right\rangle_{c}$ as a power series in the small $q$ expansion. It turns out that the result is nicely organized in terms of the variables

$$
\lambda_{i}=\frac{1}{2} q^{2} \beta_{i} \quad(i=1, \cdots, n) .
$$

\footnotetext{
${ }^{8}$ The data of $\mathcal{F}_{g}^{\text {con }}$ up to $g=20$ are available upon request to the authors.
} 
For instance, the one-point function is expanded as

$$
\langle Z(\beta)\rangle=\frac{1}{\sqrt{4 \pi \lambda}} \sum_{n, m=0}^{\infty} \nu^{-(2 n-1)} q^{2 m} \mathcal{Z}_{n, m}(\lambda) .
$$

Using the result of free energy $F_{g}$ obtained from the cut-and-join operator, we find the closed form expression of the first few terms of this expansion:

$$
\begin{aligned}
& \mathcal{Z}_{0,0}=e^{-\lambda}+\sqrt{\pi \lambda} \operatorname{Erf}(\sqrt{\lambda}) \\
& \mathcal{Z}_{0,1}=\frac{\lambda+1}{2 \lambda} e^{-\lambda} \\
& \mathcal{Z}_{0,2}=\left(\frac{1}{8 \lambda^{2}}+\frac{1}{8 \lambda}+\frac{1}{16}+\frac{\lambda}{4}\right) e^{-\lambda} \\
& \mathcal{Z}_{1,0}=\frac{-3 \lambda+2 \lambda^{2}}{12} e^{-\lambda} \\
& \mathcal{Z}_{1,1}=\left(\frac{5}{8} \lambda-\frac{2}{3} \lambda^{2}+\frac{1}{6} \lambda^{3}\right) e^{-\lambda}, \\
& \mathcal{Z}_{2,0}=\left(-\frac{3 \lambda^{2}}{32}+\frac{107 \lambda^{3}}{240}-\frac{29 \lambda^{4}}{120}+\frac{\lambda^{5}}{36}\right) e^{-\lambda},
\end{aligned}
$$

where $\operatorname{Erf}(z)$ denotes the error function. In the rest of this section, we will derive these results from the constitutive relations.

Taking the genus-zero term of (6.14), we find

$$
\langle Z(\beta)\rangle=\frac{\nu}{\sqrt{4 \pi \lambda}} \int_{0}^{1} d t_{0} e^{\beta u_{0}}+\mathcal{O}\left(\nu^{-1}\right),
$$

where $u_{0}$ satisfies the string equation of BGW model (6.5). Plugging the small $q$ expansion of $u_{0}$ in (6.13) into (6.63) we find

$$
\begin{aligned}
\int_{0}^{1} d t_{0} e^{\beta u_{0}} & =\int_{0}^{1} d t_{0} e^{-\frac{\lambda}{\left(1-t_{0}\right)^{2}}}\left[1+\frac{\lambda q^{2}}{\left(1-t_{0}\right)^{5}}+\mathcal{O}\left(q^{4}\right)\right] \\
& =-\sqrt{\pi \lambda}+e^{-\lambda}+\sqrt{\pi \lambda} \operatorname{Erf}(\sqrt{\lambda})+q^{2} \frac{1+\lambda}{2 \lambda} e^{-\lambda}+\mathcal{O}\left(q^{4}\right) .
\end{aligned}
$$

This reproduces the result of $\mathcal{Z}_{0, m}$ in (6.62) up to a constant shift $-\nu / 2$ of $\langle Z(\beta)\rangle$, which is related to the matrix model normalization vs. the supergravity normalization (2.1).

The genus-zero part of two-point function is given by $[22,60]$

$$
\begin{aligned}
\left\langle Z\left(\beta_{1}\right) Z\left(\beta_{2}\right)\right\rangle_{c}^{g=0} & =\left.\frac{\sqrt{\beta_{1} \beta_{2}}}{2 \pi\left(\beta_{1}+\beta_{2}\right)} e^{\left(\beta_{1}+\beta_{2}\right) u_{0}}\right|_{t_{0}=0} \\
& =\frac{\sqrt{\lambda_{1} \lambda_{2}}}{2 \pi\left(\lambda_{1}+\lambda_{2}\right)} e^{-\left(\lambda_{1}+\lambda_{2}\right)}\left[1+q^{2}\left(\lambda_{1}+\lambda_{2}\right)+\mathcal{O}\left(q^{4}\right)\right] .
\end{aligned}
$$

We have checked that this agrees with the direct calculation using $F_{g}$. More generally, using the genus-zero part of the KdV flow (3.23)

$$
\partial_{k} u_{0}=\partial_{0} \frac{u_{0}^{k+1}}{(k+1) !},
$$


one can show that the genus-zero $n$-point function is given by

$$
\left\langle Z\left(\beta_{1}\right) \cdots Z\left(\beta_{n}\right)\right\rangle_{c}^{g=0}=\left(-\hbar \partial_{0}\right)^{n-2} \prod_{i=1}^{n} \sqrt{\frac{\beta_{i}}{2 \pi}} \frac{e^{u_{0} \sum_{i=1}^{n} \beta_{i}}}{\sum_{i=1}^{n} \beta_{i}} .
$$

This is the same as the result of the KW model corresponding to a double-scaled hermitian matrix model. Note that (6.67) is a consequence of the genus-zero KdV equation (6.66), which is common for both the KW model and the BGW model. This is the reason why (6.67) holds for the BGW model as well.

Next let us consider the genus-one part, where the genus-one free energy is given by $(6.49)$

$$
\mathcal{F}_{1}=\frac{1}{24} \log \left(\partial_{0} u_{0}\right)+\mathcal{F}_{1}^{\mathrm{vol}}
$$

By acting the boundary creation operator we obtain the genus-one part of $\langle Z(\beta)\rangle$. From (6.67) we find

$$
B\left(\beta_{1}\right) \cdots B\left(\beta_{n}\right) u_{0}=\left(-\hbar \partial_{0}\right)^{n} \prod_{i=1}^{n} \sqrt{\frac{\beta_{i}}{2 \pi}} \frac{e^{u_{0} \sum_{i=1}^{n} \beta_{i}}}{\sum_{i=1}^{n} \beta_{i}} .
$$

Then the genus-one part of the correlator

$$
\left\langle Z\left(\beta_{1}\right) \cdots Z\left(\beta_{n}\right)\right\rangle_{c}^{g=1}=B\left(\beta_{1}\right) \cdots B\left(\beta_{n}\right) \mathcal{F}_{1}
$$

is written as a combination of $B\left(\beta_{i_{1}}\right) \cdots B\left(\beta_{i_{k}}\right) u_{0}$ in (6.69). From the logarithmic form of $\mathcal{F}_{1}$ (6.68), the combinatorics is the same as the computation of connected correlators. Thus we find

$$
\left\langle\prod_{i=1}^{n} Z\left(\beta_{i}\right)\right\rangle^{g=1}=\left.\frac{1}{24} \log \left[1+\frac{1}{\partial_{0} u_{0}} \sum_{n=1}^{\infty} \sum_{i_{1}<\cdots<i_{n}} x_{i_{1}} \cdots x_{i_{n}} B\left(\beta_{i_{1}}\right) \cdots B\left(\beta_{i_{n}}\right) \partial_{0} u_{0}\right]\right|_{\mathcal{O}\left(x_{1} \cdots x_{n}\right)} .
$$

For instance, the genus-one part of the one-point function is given by

$$
\begin{aligned}
\langle Z(\beta)\rangle^{g=1} & =\frac{1}{24 \partial_{0} u_{0}} \partial_{0}\left(B(\beta) u_{0}\right) \\
& =-\frac{1}{\nu \sqrt{4 \pi \lambda}} \frac{\lambda}{12}\left(\frac{\partial_{0}^{2} u_{0}}{\partial_{0} u_{0}}+\beta \partial_{0} u_{0}\right) e^{\beta u_{0}} .
\end{aligned}
$$

We have checked that this reproduces the $\mathcal{Z}_{1, m}$ in (6.62). Plugging the on-shell value of $u_{0}$ in (6.13), one can in principle compute the correlator at genus-one (6.71) up to any order in the small $q$ expansion.

Finally, let us consider the genus-two part. The genus-two free energy (6.50) is rewritten as

$$
\mathcal{F}_{2}=\frac{1}{\partial_{0} u_{0}} \partial_{0}\left[\frac{1}{1152} \partial_{0}^{2} \log \left(\partial_{0} u_{0}\right)-\frac{1}{1920}\left(\partial_{0} \log \left(\partial_{0} u_{0}\right)\right)^{2}\right]+\mathcal{F}_{2}^{\text {vol }}
$$

By acting the boundary creation operator $B(\beta)$ the genus-two partition function is written as

$$
\begin{aligned}
B(\beta) \mathcal{F}_{2}= & -\mathcal{F}_{2}^{\operatorname{con}} B(\beta) \log \left(\partial_{0} u_{0}\right)-\frac{\partial_{0}^{2} \log \left(\partial_{0} u_{0}\right)}{960 \partial_{0} u_{0}} \partial_{0} B(\beta) \log \left(\partial_{0} u_{0}\right) \\
& -\frac{\partial_{0} \log \left(\partial_{0} u_{0}\right)}{960 \partial_{0} u_{0}} \partial_{0}^{2} B(\beta) \log \left(\partial_{0} u_{0}\right)+\frac{1}{1152 \partial_{0} u_{0}} \partial_{0}^{3} B(\beta) \log \left(\partial_{0} u_{0}\right) .
\end{aligned}
$$


Note that $B(\beta) \log \left(\partial_{0} u_{0}\right)$ is proportional to the genus-one partition function

$$
\langle Z(\beta)\rangle^{g=1}=B(\beta) \mathcal{F}_{1}=\frac{1}{24} B(\beta) \log \left(\partial_{0} u_{0}\right) .
$$

Thus we find that the genus-two partition function is written in terms of the genus-one partition function

$$
\langle Z(\beta)\rangle^{g=2}=\left(-24 \mathcal{F}_{2}^{\text {con }}-\frac{\partial_{0}^{2} \log \left(\partial_{0} u_{0}\right)}{40 \partial_{0} u_{0}} \partial_{0}-\frac{\partial_{0} \log \left(\partial_{0} u_{0}\right)}{40 \partial_{0} u_{0}} \partial_{0}^{2}+\frac{1}{48 \partial_{0} u_{0}} \partial_{0}^{3}\right)\langle Z(\beta)\rangle^{g=1} .
$$

Again one can in principle compute $\langle Z(\beta)\rangle^{g=2}$ up to any order in the small $q$ expansion using (6.13).

Note that since the genus expansion of the one-point function in JT gravity has already been calculated in [11], one can derive (6.71) and (6.76) from the corresponding bosonic JT results simply by the replacement (6.54) and the change of variables (6.43), as stated in section $6.2 .{ }^{9}$ We verified that $(6.71)$ and (6.76) are indeed in agreement with the results obtained in [11].

\section{Conclusions and outlook}

In this paper we have studied the genus expansion of JT supergravity using the relation to the BGW $\tau$-function. We found that the matrix model of JT supergravity is nothing but the BGW model with infinite number of couplings turned on with specific values $t_{k}=\gamma_{k}$ in (2.18). We have computed the genus expansion at finite RR flux $\nu$ using the cut-and-join operator and considered the one-point function $\langle Z(\beta)\rangle$ in the low temperature regime (5.1). We found that the result is expanded in terms of the Bessel functions (5.10), which is a natural generalization of the Bessel case reviewed in section 4. Next we have considered the large $\nu$ regime (1.1). It turns out that the genus zero free energy is nonzero in this regime and thus at genus-zero we can immediately write down the connected correlator $\left\langle Z\left(\beta_{1}\right) \cdots Z\left(\beta_{n}\right)\right\rangle_{c}^{g=0}$ in terms of the potential $u_{0}$ (see $(6.15)$ and (6.67)). We have found that the hard edge of the eigenvalue density $\rho_{0}(E) \sim E^{-\frac{1}{2}}$ is reproduced by carefully taking the limit $q \rightarrow 0$ (6.24). We have also found that the free energy of the BGW model satisfies the constitutive relation, i.e. the higher genus free energy is written as a combination of genus-zero quantities $\partial_{0}^{n} u_{0}(n \geq 1)$. This enables us to compute the higher genus corrections to the correlators $\left\langle Z\left(\beta_{1}\right) \cdots Z\left(\beta_{n}\right)\right\rangle_{c}$ up to any order in principle. We obtained the $g=1$ correction in the general form (6.71). In fact, the constitutive relation of the BGW model is identical to that of the KW model. This means that one can get the higher genus results immediately from the corresponding results in the bosonic JT case $[11,12]$ by the replacement $(6.54)$. We demonstrated it for the one-point function at $g=1(6.71)$ and $g=2(6.76)$ as an example.

There are several open questions. Since the genus expansion of JT supergravity is an asymptotic series we expect that there appear non-perturbative effects which are analogue

\footnotetext{
${ }^{9}$ One also needs to change the overall sign of the one-point function because the boundary creation operator (2.13) in JT supergravity has an extra overall sign factor as compared to the JT case.
} 
of the D-branes in minimal superstring theory. It would be interesting to clarify the spacetime picture of these non-perturbative effects. It would also be interesting to generalize our computations to other Altland-Zirnbauer ensembles studied in [16] and consider JT (super)gravity on unorientable surfaces along the lines of our work. Finally, we would like to understand the physical origin of the constitutive relation more clearly. The universality of this relation means that bosonic JT gravity and supersymmetric JT gravity are both characterized by the same form of the free energy of topological gravity. For this relation to work, it is essential to consider the large $\nu$ regime (1.1) whose meaning is not well understood in the mathematics literature, although this limit is natural from the viewpoint of physics [35]. It would be interesting to clarify this point further.

\section{Acknowledgments}

We would like to thank Clifford V. Johnson for correspondence. This work was supported in part by JSPS KAKENHI Grant Nos. 19K03845 and 19K03856, and JSPS Japan-Russia Research Cooperative Program.

Open Access. This article is distributed under the terms of the Creative Commons Attribution License (CC-BY 4.0), which permits any use, distribution and reproduction in any medium, provided the original author(s) and source are credited.

\section{References}

[1] R. Jackiw, Lower Dimensional Gravity, Nucl. Phys. B 252 (1985) 343 [InSPIRE].

[2] C. Teitelboim, Gravitation and Hamiltonian Structure in Two Space-Time Dimensions, Phys. Lett. B 126 (1983) 41 [inSPIRE].

[3] A. Almheiri and J. Polchinski, Models of AdS 2 backreaction and holography, JHEP 11 (2015) 014 [arXiv: 1402.6334] [INSPIRE].

[4] J. Maldacena, D. Stanford and Z. Yang, Conformal symmetry and its breaking in two dimensional Nearly Anti-de-Sitter space, PTEP 2016 (2016) 12C104 [arXiv:1606.01857] [INSPIRE].

[5] J. Engelsöy, T.G. Mertens and H. Verlinde, An investigation of $A d S_{2}$ backreaction and holography, JHEP 07 (2016) 139 [arXiv: 1606. 03438] [INSPIRE].

[6] D. Harlow and D. Jafferis, The Factorization Problem in Jackiw-Teitelboim Gravity, JHEP 02 (2020) 177 [arXiv: 1804.01081] [INSPIRE].

[7] P. Saad, S.H. Shenker and D. Stanford, JT gravity as a matrix integral, arXiv:1903.11115 [INSPIRE].

[8] B. Eynard and N. Orantin, Weil-Petersson volume of moduli spaces, Mirzakhani's recursion and matrix models, arXiv:0705.3600 [INSPIRE].

[9] M. Mirzakhani, Simple geodesics and Weil-Petersson volumes of moduli spaces of bordered Riemann surfaces, Invent. Math. 167 (2006) 179 [INSPIRE].

[10] B. Eynard and N. Orantin, Invariants of algebraic curves and topological expansion, Commun. Num. Theor. Phys. 1 (2007) 347 [math-ph/0702045] [INSPIRE]. 
[11] K. Okuyama and K. Sakai, JT gravity, KdV equations and macroscopic loop operators, JHEP 01 (2020) 156 [arXiv: 1911.01659] [INSPIRE].

[12] K. Okuyama and K. Sakai, Multi-boundary correlators in JT gravity, JHEP 08 (2020) 126 [arXiv:2004.07555] [INSPIRE].

[13] P. Zograf, On the large genus asymptotics of Weil-Petersson volumes, arXiv:0812.0544 [INSPIRE].

[14] M. Kontsevich, Intersection theory on the moduli space of curves and the matrix Airy function, Commun. Math. Phys. 147 (1992) 1 [INSPIRE].

[15] E. Witten, Two-dimensional gravity and intersection theory on moduli space, Surveys Diff. Geom. 1 (1991) 243.

[16] D. Stanford and E. Witten, JT Gravity and the Ensembles of Random Matrix Theory, arXiv: 1907.03363 [INSPIRE].

[17] P. Norbury, Enumerative geometry via the moduli space of super Riemann surfaces, arXiv:2005.04378 [INSPIRE].

[18] E. Witten, Volumes and Random Matrices, arXiv:2004.05183 [INSPIRE].

[19] A. Alexandrov, Cut-and-join description of generalized Brezin-Gross-Witten model, Adv. Theor. Math. Phys. 22 (2018) 1347 [arXiv:1608.01627] [INSPIRE].

[20] R. Dijkgraaf and E. Witten, Mean Field Theory, Topological Field Theory, and Multimatrix Models, Nucl. Phys. B 342 (1990) 486 [inSPIRE].

[21] T. Eguchi, Y. Yamada and S.-K. Yang, On the genus expansion in the topological string theory, Rev. Math. Phys. 7 (1995) 279 [hep-th/9405106] [INSPIRE].

[22] P.H. Ginsparg and G.W. Moore, Lectures on $2-D$ gravity and $2-D$ string theory, in Theoretical Advanced Study Institute (TASI 92): From Black Holes and Strings to Particles, pp. 277-469, 10, 1993 [hep-th/9304011] [INSPIRE].

[23] A. Altland and M.R. Zirnbauer, Nonstandard symmetry classes in mesoscopic normal-superconducting hybrid structures, Phys. Rev. B $5 \mathbf{5}$ (1997) 1142 [cond-mat/9602137] [INSPIRE].

[24] P. Norbury, A new cohomology class on the moduli space of curves, arXiv:1712.03662.

[25] D.J. Gross and E. Witten, Possible Third Order Phase Transition in the Large $N$ Lattice Gauge Theory, Phys. Rev. D 21 (1980) 446 [INSPIRE].

[26] E. Brézin and D.J. Gross, The External Field Problem in the Large $N$ Limit of QCD, Phys. Lett. B 97 (1980) 120 [INSPIRE].

[27] D.J. Gross and M.J. Newman, Unitary and Hermitian matrices in an external field. 2: The Kontsevich model and continuum Virasoro constraints, Nucl. Phys. B 380 (1992) 168 [hep-th/9112069] [INSPIRE].

[28] A. Mironov, A. Morozov and G.W. Semenoff, Unitary matrix integrals in the framework of generalized Kontsevich model. 1. Brezin-Gross-Witten model, Int. J. Mod. Phys. A 11 (1996) 5031 [hep-th/9404005] [INSPIRE].

[29] M. Mulase and B. Safnuk, Mirzakhani's recursion relations, Virasoro constraints and the KdV hierarchy, math/0601194 [INSPIRE]. 
[30] R. Dijkgraaf and E. Witten, Developments in Topological Gravity, Int. J. Mod. Phys. A 33 (2018) 1830029 [arXiv: 1804.03275] [INSPIRE].

[31] N. Do and P. Norbury, Topological recursion on the Bessel curve, Commun. Num. Theor. Phys. 12 (2018) 53 [arXiv: 1608.02781] [InSPIRE].

[32] T.R. Morris, 2-D quantum gravity, multicritical matter and complex matrices, Report number: FERMILAB-PUB-90-136-T (1990).

[33] S. Dalley, C.V. Johnson and T.R. Morris, Multicritical complex matrix models and nonperturbative $2-D$ quantum gravity, Nucl. Phys. B 368 (1992) 625 [INSPIRE].

[34] S. Dalley, C.V. Johnson, T.R. Morris and A. Watterstam, Unitary matrix models and $2-D$ quantum gravity, Mod. Phys. Lett. A 7 (1992) 2753 [hep-th/9206060] [INSPIRE].

[35] I.R. Klebanov, J.M. Maldacena and N. Seiberg, Unitary and complex matrix models as $1-D$ type 0 strings, Commun. Math. Phys. 252 (2004) 275 [hep-th/0309168] [INSPIRE].

[36] C.V. Johnson, Nonperturbative Jackiw-Teitelboim gravity, Phys. Rev. D 101 (2020) 106023 [arXiv: 1912.03637] [INSPIRE].

[37] C.V. Johnson, JT Supergravity, Minimal Strings, and Matrix Models, arXiv:2005.01893 [INSPIRE].

[38] C.V. Johnson, Explorations of Non-Perturbative JT Gravity and Supergravity, arXiv: 2006.10959 [INSPIRE].

[39] B. Dubrovin, D. Yang and D. Zagier, On tau-functions for the KdV hierarchy, arXiv: 1812.08488 [INSPIRE].

[40] M. Fukuma, H. Kawai and R. Nakayama, Continuum Schwinger-dyson Equations and Universal Structures in Two-dimensional Quantum Gravity, Int. J. Mod. Phys. A 6 (1991) 1385 [INSPIRE].

[41] R. Dijkgraaf, H.L. Verlinde and E.P. Verlinde, Loop equations and Virasoro constraints in nonperturbative $2-D$ quantum gravity, Nucl. Phys. B 348 (1991) 435 [INSPIRE].

[42] C.V. Johnson, On integrable $c<1$ open string theory, Nucl. Phys. B 414 (1994) 239 [hep-th/9301112] [INSPIRE].

[43] M. Mirzakhani and P. Zograf, Towards large genus asymtotics of intersection numbers on moduli spaces of curves, arXiv:1112.1151 [INSPIRE].

[44] K. Okuyama and K. Sakai, Resurgence analysis of $2 d$ Yang-Mills theory on a torus, JHEP 08 (2018) 065 [arXiv: 1806.00189] [INSPIRE].

[45] T. Nagao and K. Slevin, Nonuniversal correlations for random matrix ensembles, J. Math. Phys. 34 (1993) 2075.

[46] M. Bertola and G. Ruzza, Brezin-Gross-Witten tau function and isomonodromic deformations, Commun. Num. Theor. Phys. 13 (2019) 827 [arXiv:1812.02116] [InSPIRE].

[47] A. Cherman, P. Koroteev and M. Ünsal, Resurgence and Holomorphy: From Weak to Strong Coupling, J. Math. Phys. 56 (2015) 053505 [arXiv: 1410.0388] [INSPIRE].

[48] T. Banks, M.R. Douglas, N. Seiberg and S.H. Shenker, Microscopic and Macroscopic Loops in Nonperturbative Two-dimensional Gravity, Phys. Lett. B 238 (1990) 279 [INSPIRE].

[49] A.M. García-García and J.J.M. Verbaarschot, Spectral and thermodynamic properties of the Sachdev-Ye-Kitaev model, Phys. Rev. D 94 (2016) 126010 [arXiv:1610.03816] [INSPIRE]. 
[50] J.S. Cotler et al., Black Holes and Random Matrices, JHEP 05 (2017) 118 [Erratum ibid. 09 (2018) 002] [arXiv: 1611.04650] [INSPIRE].

[51] P. Saad, S.H. Shenker and D. Stanford, A semiclassical ramp in SYK and in gravity, arXiv: 1806.06840 [INSPIRE].

[52] S. Sachdev and J. Ye, Gapless spin fluid ground state in a random, quantum Heisenberg magnet, Phys. Rev. Lett. 70 (1993) 3339 [cond-mat/9212030] [INSPIRE].

[53] A. Kitaev, A simple model of quantum holography (part 1 and 2), talks at KITP on 7 April: http://online.kitp.ucsb.edu/online/entangled15/kitaev/, and 27 May: http://online.kitp.ucsb.edu/online/entangled15/kitaev2/, University of California, Santa Barbara U.S.A. (2015).

[54] C. Itzykson and J.B. Zuber, Combinatorics of the modular group. 2. The Kontsevich integrals, Int. J. Mod. Phys. A 7 (1992) 5661 [hep-th/9201001] [INSPIRE].

[55] H. Maxfield and G.J. Turiaci, The path integral of $3 D$ gravity near extremality; or, JT gravity with defects as a matrix integral, arXiv:2006.11317 [INSPIRE].

[56] E. Witten, Matrix Models and Deformations of JT Gravity, arXiv:2006.13414 [INSPIRE].

[57] J. Zhou, On Topological 1D Gravity. I, arXiv:1412.1604 [INSPIRE].

[58] Q. Zhang and J. Zhou, On Itzykson-Zuber Ansatz, JHEP 09 (2019) 075 [arXiv:1904.09071] [INSPIRE].

[59] H. Ooguri and C. Vafa, World sheet derivation of a large $N$ duality, Nucl. Phys. B 641 (2002) 3 [hep-th/0205297] [INSPIRE].

[60] G.W. Moore, N. Seiberg and M. Staudacher, From loops to states in $2-D$ quantum gravity, Nucl. Phys. B 362 (1991) 665 [InSPIRE]. 